University of Denver

Digital Commons @ DU

$1-1-2019$

\title{
Small-Scale Microgrid Energy Market Based on PILT-DAO
}

Tianlu Gao

University of Denver

Follow this and additional works at: https://digitalcommons.du.edu/etd

Part of the Electrical and Computer Engineering Commons

\section{Recommended Citation}

Gao, Tianlu, "Small-Scale Microgrid Energy Market Based on PILT-DAO" (2019). Electronic Theses and Dissertations. 1578.

https://digitalcommons.du.edu/etd/1578

This Thesis is brought to you for free and open access by the Graduate Studies at Digital Commons @ DU. It has been accepted for inclusion in Electronic Theses and Dissertations by an authorized administrator of Digital Commons@DU. For more information, please contact jennifer.cox@du.edu,dig-commons@du.edu. 
SMALL-SCALE MICROGRID ENERGY

MARKET BASED ON PILT-DAO

\author{
A Thesis \\ Presented to \\ the Faculty of the Daniel Felix Ritchie School \\ of Engineering and Computer Science \\ University of Denver
}

In Partial Fulfillment

of the Requirements for the Degree

Master of Science

by

Tianlu Gao

June 2019

Advisor: Wenzhong Gao 
(C)Copyright by Tianlu Gao 2019

All Rights Reserved 
Author: Tianlu Gao

Title: SMALL-SCALE MICROGRID ENERGY MARKET BASED ON PILT-

DAO

Advisor: Wenzhong Gao

Degree Date: June 2019

\section{Abstract}

With the installed capacities of Distributed Generations (DGs) dramatically increasing in power systems from Distributed Energy Resources (DERs) such as hydropower, wind, solar, geothermal and biomass, the operation methods of DERs tradings or transactions become more and more complicated. However, the energy market of DERs in Microgrids (MGs) is still under devolvement due to low security and transparency at present. Therefore, a small-scale microgrid energy market is proposed in this study based on Decentralized Autonomous Organization of Parallel, Integrity, Longevity, and Transparency (PILT-DAO) of the features of the blockchain. The microgrid owners can complete the transaction in the PILT-DAO market.

In order to implement this energy trading platform, the first step is to simulate a modified distributed IEEE 13 node test feeders system. The next step is to develop a price mechanism method based on a consensus + innovation distributed algorithm to calculate the distributed Distribution Locational Marginal Price (DLMP). At the meantime, smart meters record the Power Flow (PF) data of each DG as one node of the whole simulated distributed power system and send them to blockchain including distributed price and power generation data. The third step is to constitute a decentralized autonomous market by programming smart contracts in Ethereum 
DAO, running in an artificial system parallelly. A case study of a small-scale microgrid energy market based on PILT-DAO is illustrated followed by the conclusion. 


\section{Acknowledgements}

I would like to extend thanks to the many people who so generously supports to the work presented in my thesis.

Special thanks go to my enthusiastic supervisor, Dr. Jason Jun Zhang. My graduate life has been an amazing experience and I thank him again wholeheartedly, not only for his tremendous academic support but also for giving me so many wonderful opportunities.

Similarly, profound gratitude goes to Prof. David Wenzhong Gao, who has truly shared his taxonomic expertise so willingly as a mentor. I would like to thank sepcially Dr. Yingchen Zhang for his mentorship and internship at Natinal Rereable Energy Laboratory. In addition, special thanks go to Dr. Yun-bo Yi, Dr. Amin Khodaei, Dr. Ronald Delyser, and Dr. Yin Yao for their great lectures.

Moreover, I am also hugely indebted to my labmates and friends at the University of Denver: Huaiguang Jiang, Jun Hao, Yi Gu, Xiaoxiao Dai, Yishi Lee, Abdulaziz Almalaq, Weihang Yan, Wei Gao, Qiao Li, Jian Qiu, Qi Pan, and Zhou Chen, for spending amazing time together.

Last but not the least, thanks go to my family, my wife (Xuan He), my mother (Xiaoqin Ouyang), my father (Wenxing Gao) and my sister (Bolan Gao), for unbelievable support. They are the most important people in my life and I dedicate this thesis to them. 


\section{Table of Contents}

Acknowledgement $\ldots \ldots \ldots \ldots \ldots \ldots$ iv

List of Figures $\ldots \ldots \ldots \ldots \ldots$ vi

1 Introduction 1

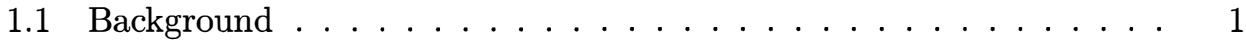

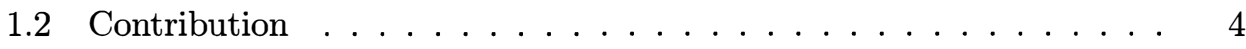

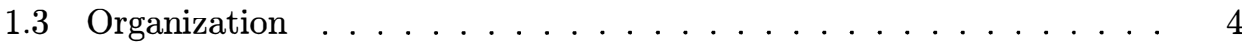

2 Literature Review 6

2.1 Bitcoin and Blockchain . . . . . . . . . . . . . . 6

2.2 Smart Contract . . . . . . . . . . . . . . . 7

2.3 Microgrid . . . . . . . . . . . . . . . . 9

2.4 Parallel System Theory and ACP Approach . . . . . . . . . . . . 9

2.5 Social Energy . . . . . . . . . . . . . . . . . . . . . 11

2.6 Distribution Locational Marginal Price . . . . . . . . . . . . . . 11

3 Entities Modeling Layer $\quad 13$

4 Price Mechanism Layer $\quad 18$

5 PILT-DAO Market Layer 31

5.1 Whole PILT-DAO Blueprint . . . . . . . . . . . . . . . 31

5.2 Case Study . . . . . . . . . . . . . . . . . 34

6 Conclusion and Future Works $\quad 39$

$\begin{array}{ll}\text { Bibliography } & 40\end{array}$

$\begin{array}{lr}\text { Appendices } & 45\end{array}$

A ACRONYMS $\quad 45$

$\begin{array}{ll}\text { B Nomenclature } & 47\end{array}$ 


\section{List of Figures}

1 Introduction

1.1 Architecture of small-scale microgrid energy market . . . . . . . 5

2 Literature Review

2.1 The flow chart of all processes of transactions in blockchain $\ldots \ldots .7$

2.2 The DAO business model . . . . . . . . . . . . . 8

2.3 A conceptual framework of PILT-DAO . . . . . . . . . . . . . 10

2.4 PILT-DAO based on ACP: from CPS to CPSS . . . . . . . . . . 11

\section{Entities Modeling Layer}

3.1 Modified IEEE 13 node test feeder system topology . . . . . . . . . . 14

3.2 Load input profile . . . . . . . . . . . . . . . . . . 17

\section{Price Mechanism Layer}

4.1 Consensus + innovations approach flow chart . . . . . . . . . 19

4.2 DCOPF consensus + innovations approach flow chart . . . . . . . 24

4.3 Generation units output . . . . . . . . . . . . . 25

4.4 Generation units output for congested case . . . . . . . . . 25

4.5 Distributed DLMPs . . . . . . . . . . . . . . . . 26

4.6 Distributed DLMPs for congested case . . . . . . . . . . . . . 26

4.7 The 1000th iteration of distributed DLMPs at hour 1, 10, 14, and $18 \quad 27$

4.8 The 1000th iteration of generation output at hour 1, 10, 14, and 18 . 28

4.9 The 1000th iteration of distributed DLMPs in the congested case . . 29

4.10 The 1000th iteration of generation output in the congested case . . . 30

\section{PILT-DAO Market Layer}

5.1 Whole PILT-DAO layers blueprint . . $\quad 32$

5.2 The flowchart of detailed parallel execution process in PILT-DAO . . 33

5.3 Distributed power trading system architecture . . . . . . . . . . 34

5.4 The home page of distribution power trading platform . . . . . . . 35

5.5 The block page of distribution power trading platform . . . . . . 36

5.6 The transaction page of distribution power trading platform . . . . 36

5.7 The transaction page of Ethereum Geth platform . . . . . . . . . . 37

5.8 The transactions information in Ethereum Geth platform . . . . . . 38

5.9 The home page of distribution power trading platform after transactions 38 


\section{Chapter 1}

\section{Introduction}

\section{$1.1 \quad$ Background}

In 1927, the Pennsylvania-New Jersey Interconnects became the first U.S. power pool, transitioning to a fully independent transmission organization in 1997 with the opening of its first bid-based energy market. Federal Energy Regulatory Commission (FERC) approved the Pennsylvania, New Jersey, Maryland (PJM) pool as the first Independent System Operator (ISO) that year. And, two decades ago from now, a lot of studies show that electric utility companies quickly realized that they could interconnect with one another to decrease costs and enhance reliability and security, such as reported in Western Wind and Solar Integration Study and Eastern Renewable Generation Integration $[1,2]$. Thus, they began to share generation resources in "power pools". As a result, the utility can transfer power to another via either wholesale or retail transactions.

Over time, electricity is widely regarded as a commodity. As a commodity, electricity is bought and sold as power (measured in KiloWatts or MegaWatts) and energy (measured in KiloWatt-hours) with various attributes being traded in electricity markets. The importance of transparency in wholesale electricity markets 
was underscored by the Energy Policy Act of 2005, which aimed to facilitate price transparency in interstate markets for the sale and transmission of electric energy, and to prohibit energy market manipulation. However, under FERC regulatory jurisdiction, each Regional Transmission Organization (RTO) has developed its own regulations or rules on markets. These regulations and rules let operational issues and regional differences more and more difficult. Electricity market issues can be usually separated into two categories: manipulation by market participants or RTO market structural issues. According to a report on electricity markets in 2016 from Congressional Research Service (CRS), which is a federal legislative branch agency and housed inside the Library of Congress and charged with providing the United States Congress non-partisan advice on issues that may come before Congress, competitive electricity markets should be developed by restructuring utility and RTOs [3].

Throughout the history of electricity markets in the United States, the centralized electricity markets of renewable energy sources are recently playing an important role in energy generation markets. However, in existing centralized microgrid energy markets, buyers or consumers can only indirectly trade with generation suppliers through the retailers or utility companies. Obviously, direct trading between consumers and generation suppliers will both improve their own benefits without retailers as an inter mediator or third party. However, the transaction management methods of centralized electricity markets are still facing the following major problems: $[4,5,6,7,8,9,10,11]$

- DG suppliers need to pay high operation and regulation fees for transactions, and prices are decided by the centralized energy market;

- Centralized electricity markets face information security problem such as losing data and users' privacy; 
- It is difficult to ensure integrity, longevity, and transparency of transaction information between two parties with 100 percent trust.

Therefore, we need an efficient way to create a motivation for improving the benefits of renewable energy for both sides. In this thesis, a new energy market is proposed to design with new technologies for solving those problems.

The new concept of blockchain was introduced by Satoshi Nakamoto in his paper entitled "Bitcoin: A Peer-to-Peer Electronic Cash System" in 2009 for solving double-spending problem without a third central party such as retailer or utility company in microgrid energy markets, using the technologies of asymmetric encryption, digital signature, and consensus mechanism [12]. According to Satoshi's essay, a blockchain is a distributed peer to peer (p2p) ledger with high Byzantine fault tolerance. And it links and records the hash number of the previous block, a timestamp and transaction data. Because the information is noted in each block with timestamp and transaction data by using peer to peer network, the blockchains possess some properties such as decentralization, better security, and consistency, etc.

In addition, Decentralized Autonomous Organization(DAO) was introduced by Vitalik Buterin who is a co-founder of the most popular public blockchain platform Ethereum into blockchains [13]. The definition was explained as "it is an entity that lives on the internet and exists autonomously, but also heavily relies on hiring individuals to perform certain tasks that the automaton itself cannot do" [14]. Therefore, DAO aims to be a platform of integrity, longevity, and transparency where all people can manage their own identity, data, and asset on a blockchain.

A controversial crowdfunding project named "The DAO" was started in May 2016 which provides a new decentralized business model by programming a set of contracts on Ethereum public blockchain. However, it was hacked in June 2016 [15]. Obviously, the business model has a grand challenge to be a successful decentralized 
autonomous organization without any testing operations at the same time in another "parallel chain". Therefore, we consider applying another useful theory, parallel system methodology, in establishing this ILT-DAO business model. Specifically, PILT-DAO is based on parallel system methodology and the ACP approaches, i.e., modeling with "artificial systems" (A), analyzing with "computational experiments" (C), and controlling through "parallel execution" (P) $[16,17]$.

\subsection{Contribution}

According to above transaction problems of existing grid market and the advantages of PILT-DAO, this thesis proposes a future energy market based on PILTDAO, which is illustrated in Figure 1.1 "architecture of small-scale microgrid energy market," to solve those problems - high operation cost, low security, and low transparency. Therefore, this architecture is designed with three layers including entities

modeling layer, pricing mechanism layer, and PILT-DAO market layer to update previous three layers such as distributed energy resource layer, distribution system operator layer and regional transmission organization layer.

\subsection{Organization}

Chapter 1 of this thesis is the introduction. Chapter 2 presents the literature reviews. Chapter 3 describes the modeling of modified IEEE 13 nodes test feeder system. Chapter 4 depicts how a consensus + innovation approach achieves distributed multi-agent coordination in a Microgrid and calculate the DLMPs of this test power system for this energy market. Chapter 5 demonstrates a study case to program smart contracts into PILT-DAO communicating with calculated distributed DLMPs and power generation data in previous chapter. Chapter 6 concludes this thesis and discusses the future works. 


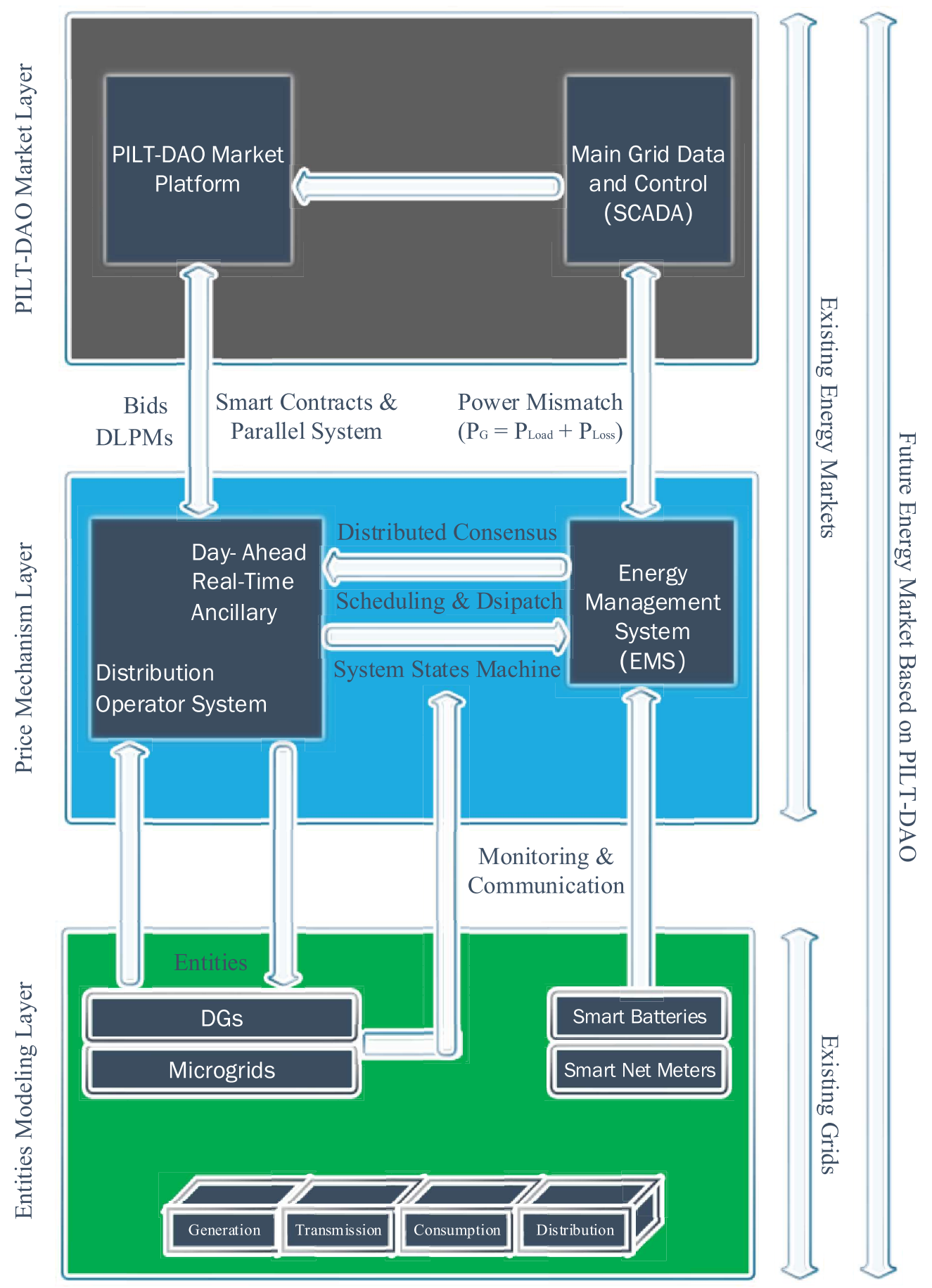

Figure 1.1: Architecture of small-scale microgrid energy market 


\section{Chapter 2}

\section{Literature Review}

\subsection{Bitcoin and Blockchain}

Before the system of Bitcoin appeared, digital signatures have solved the problems in a $\mathrm{p} 2 \mathrm{p}$ electronic cash system, in which money sender can directly pay to another without a third party such as a financial institute. However, it still needs the support of a third trusted authority to prevent double-spending. As a result, such systems do not have absolute integrity, longevity, and transparency because of the existence of third parties.

After creating the concept of the blockchain, the above-mentioned problems are solved by using hashing, timestamps, Proof-of-Work(PoW), and Merkle tree, etc. The flow chart of all processes of transactions in the bitcoin system is shown in Figure 2.1 "the flow chart of all processes of transactions in the blockchain" [12].

That begins with a timestamp server on the block of hash, and each timestamp includes the previous timestamp in its block of hash to constitute a chain such as an issue number of IEEE transaction. And then, adding a "Nonce Number" in the block implements the PoW, which gives the hash of block the required zero bits until a value is matched. That means a CPU has satisfied the PoW, and the 
new transactions are broadcast to the longest PoW chain of the whole peer-to-peer network. Therefore, the block cannot be altered. Next block will be chained after it. If an attacker would like to modify a previous block in this chain, he/she needs to redo the PoW of all blocks after the changed block and exceed the work of the honest nodes. Finally, this blockchain system achieves a peer-to-peer electronic cash system without a third party and prevents double-spending [12].

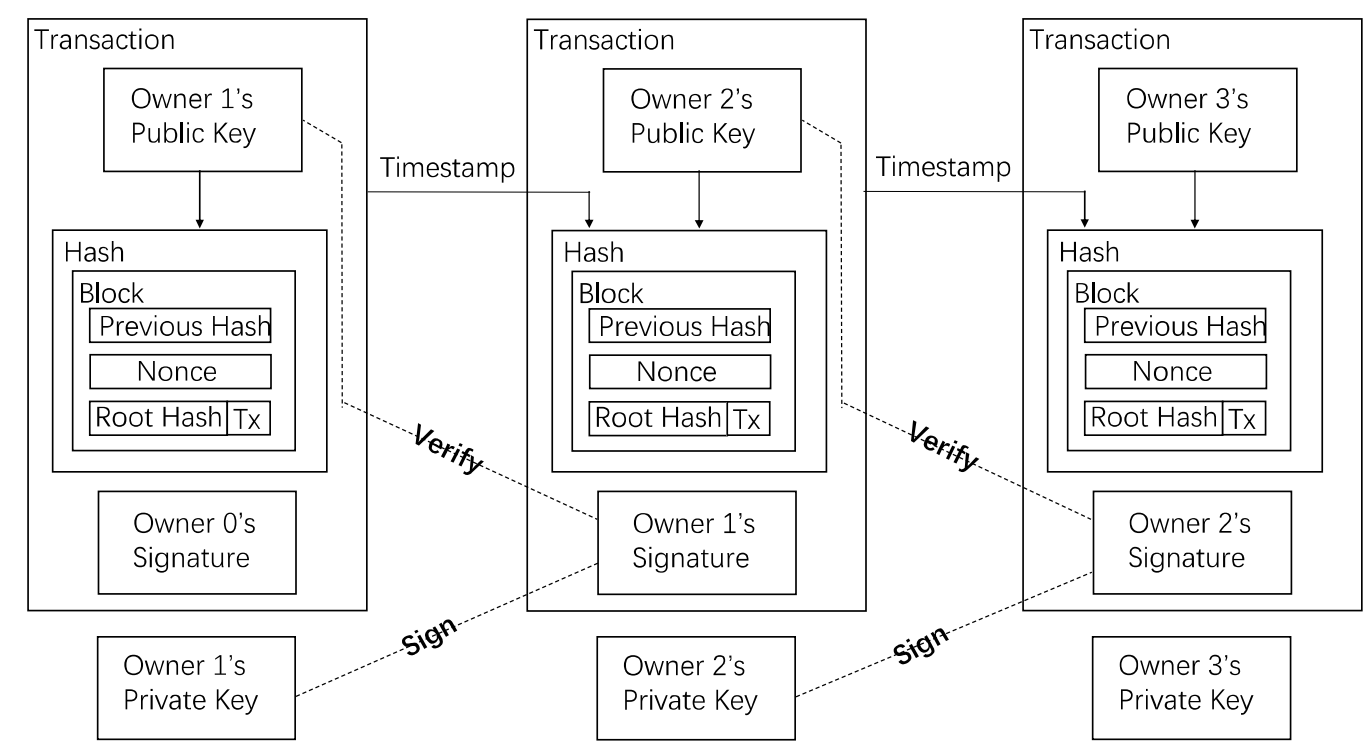

Figure 2.1: The flow chart of all processes of transactions in blockchain [12]

\subsection{Smart Contract}

A smart contract is a contract that is programmed in computers as a protocol and allows us to make sets of credible transactions without third parties. It was proposed by Nick Szabo in 1994 [18]. The goals of creating smart contracts are to enhance security and to decrease transaction costs as compared to existing transaction systems. Currently, some cryptocurrencies have embedded smart contracts in their own blockchain ecological systems. Among them, Ethereum is one of the 
most successful and distinguished platforms to implement smart contracts and a new token system.

Furthermore, a new business model is born with blockchain: The DAO. The DAO is an unstopped blockchain, run and written by sets of smart contracts to achieve an autonomy system. The DAO business model is shown in Figure 2.2. In this figure, all participants are witnesses in the blockchain ecological system, and they can trade energy by smart contracts in the PILT-DAO energy market based on blockchain network. For example, Alice sends a detailed smart contract of buying energy to Bob such as period, amount, and price, etc.. And then, after Bob confirmed the smart contract and Alice's historical credibility, he sells the energy via physical devices according to this contract. Finally, the transaction is done and recorded by blockchain system $[15,19]$.

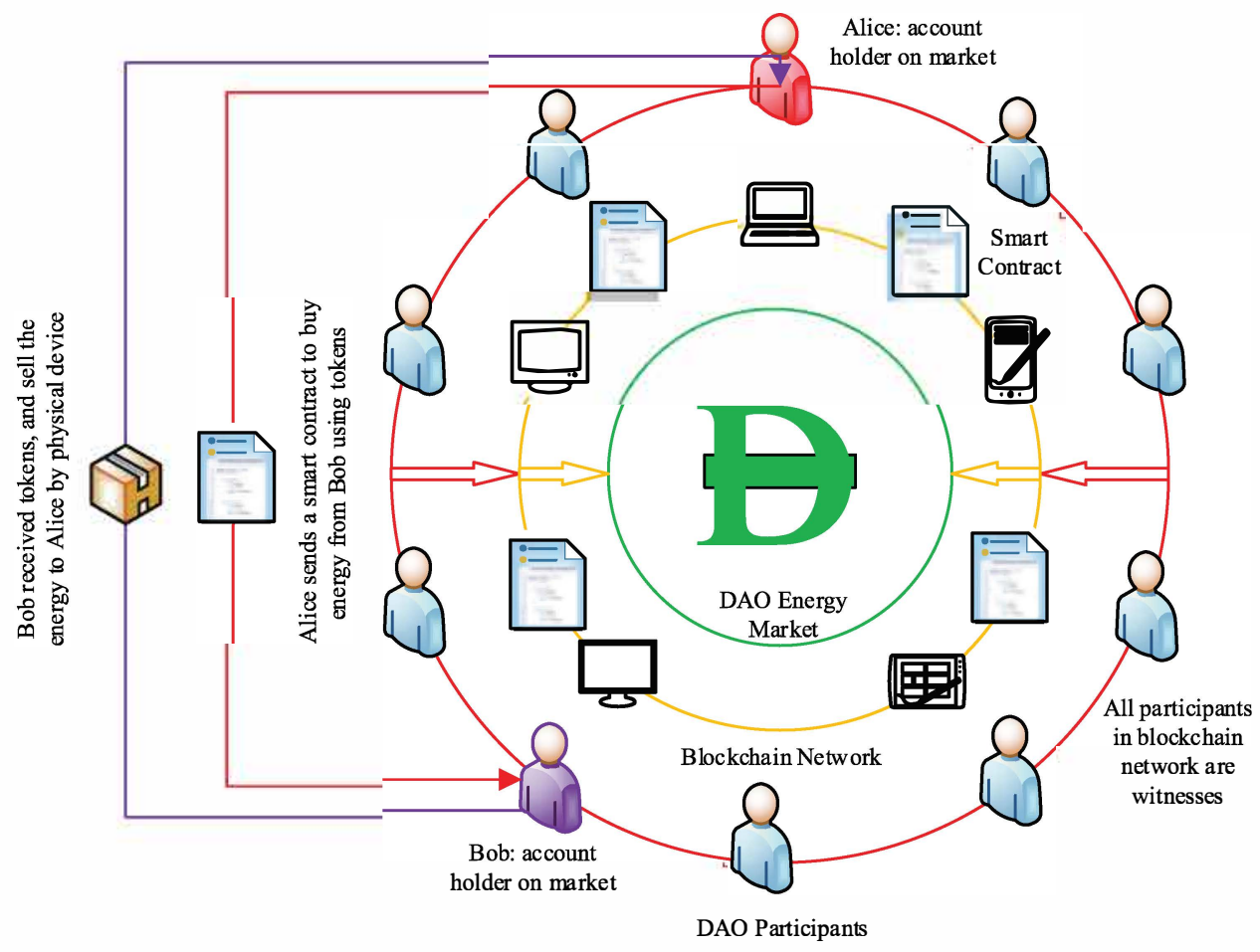

Figure 2.2: The DAO business model 


\subsection{Microgrid}

A Microgrid (MG) is considered as a distributed energy system, which consists of Distributed Generators (DGs), Distributed Storages (DSs), and local loads. It provides a solution to manage local generations and loads as a single grid-level entity. Campus institution, remote "off-grid", military base, and commercial and industrial microgrids are the four typical kinds. Generally, a MG can be operated in two modes such as grid-connected and islanded mode.

Moreover, there are several advantages of MGs in two modes. Under the gridconnected mode, ancillary services can be provided by trading activity. And the real and reactive power generated by MGs should be in balance with the demand of local loads in the islanded mode. However, the methods of control and protection are the two main challenges for MGs such as bidirectional power flow, stability issues, and low inertia, etc. Also, with various renewable energies, modern power systems such as transmission and distribution power systems are integrated with a lot of new technologies [20, 21, 22, 23].

\subsection{Parallel System Theory and ACP Approach}

The key principle of the parallel system theory is to correspond with the actual system (a system of real users and physical devices) for setting up one or more artificial systems (a system of software-defined users and devices) in the cyberspace. In this thesis, the artificial ILT-DAO system is managed, evaluated, and trained to implement parallel execution with the actual ILT-DAO system. A conceptual framework of PILT-DAO is shown in Figure 2.3. As a result, an energy market based on PILT-DAO is created.

To date, ACP is the best approach to achieve a parallel system theory [17]. There are three major steps. Firstly, one or more artificial systems are base on learning and 
training from the actual system (learning and training). Secondly, experimenting and evaluating the artificial systems reflects the status and evolution pattern of the actual system (Experiment and evaluation). Thirdly, the parallel execution between actual and artificial systems achieves the management and control of the real physical system in real-time (Management and control).

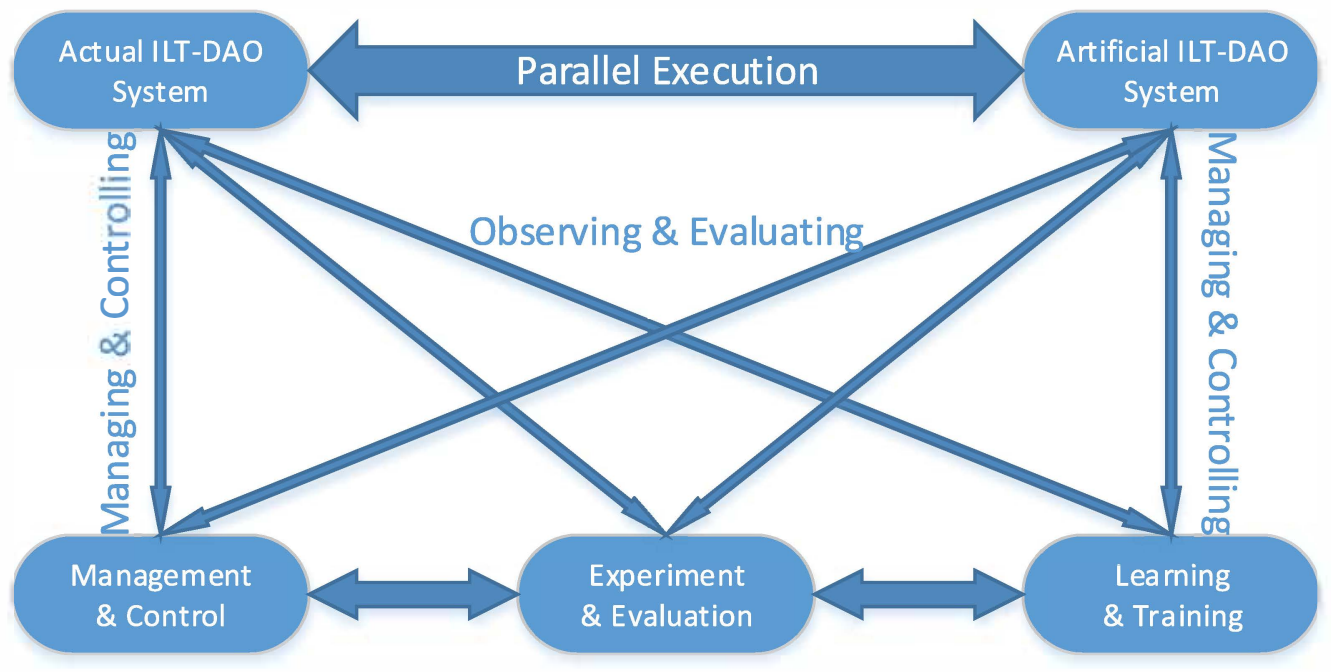

Figure 2.3: A conceptual framework of PILT-DAO

Based on the ACP approach, the PILT-DAO can be defined as an ILT-DAO which is born from the interactions and parallel executions (depiction, prediction, and conduction) between real physical and software-defined systems.

However, after human interaction getting involved in, the cyber-physical-social system (CPSS) will be established by parallel system theory instead of previous cyber-physical system (CPS). The Newtons Laws (Small Data, Big Laws) cannot be applied to CPSS straightly, thus, the Mertons Laws (Big Data, Small Laws) should be exploited [24]. PILT-DAO systems as a new paradigm based on ACP must be applied to this new technology. The whole processes are shown in Figure 2.4 . 


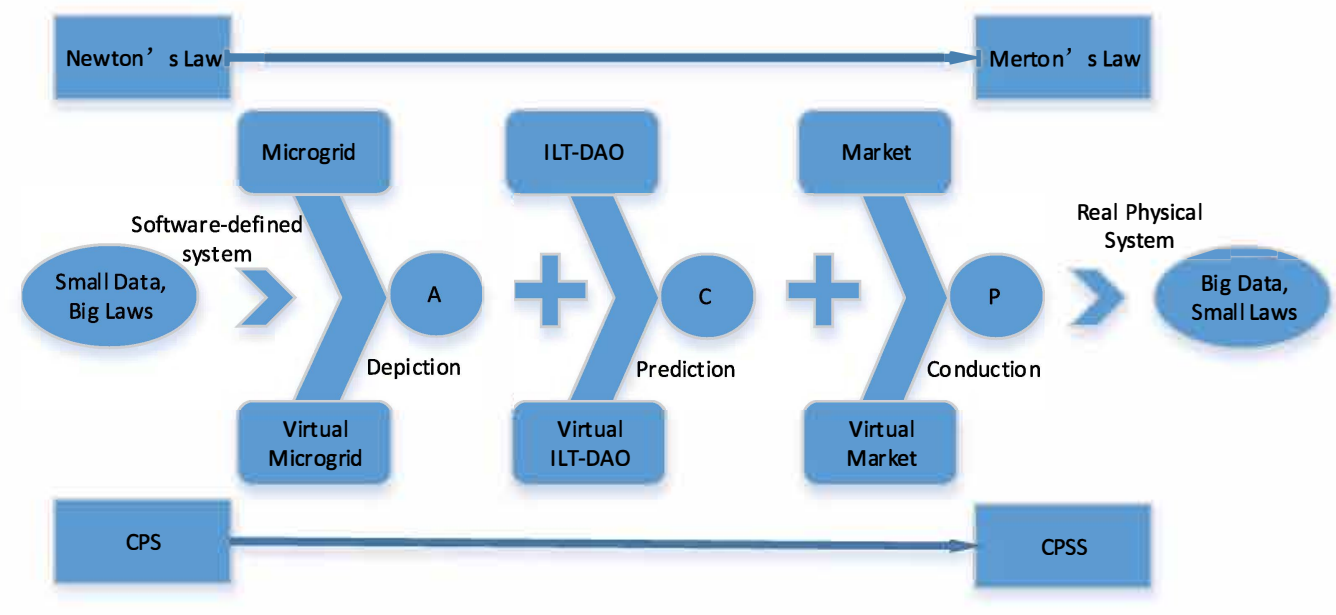

Figure 2.4: PILT-DAO based on ACP: from CPS to CPSS

\subsection{Social Energy}

The definition of social energy is introduced by Jun Jason Zhang et al in a pa-

per, "Social energy: mining energy from the society" [25]. A social energy system is a complex physical energy system, which is enabled by the recent advancement in intelligent technology, including artificial intelligence, machine learning, sensing, communication, Internet of Things technologies, and massive high-performance computing and extreme-scale data analytics technologies. In this paper, authors provide power system simulation, real-time pricing at distribution level, and social response to the pricing signals.

\subsection{Distribution Locational Marginal Price}

Distribution Locational Marginal Prices (DLMPs) is a mathematical method for setting the wholesale electric energy prices and for reflecting the value of electric energy at different locations in a distribution system corresponding to Locational Marginal Price (LMP) in a transmission system [26]. PJM learning center explains that LMP is analogous to a taxi ride for megawatts of electricity. When traffic is 
light, you can expect a consistent and predictable taxi fee, which would represent a period with little to no congestion on the grid. Similarly, heavy traffic results in a higher fee, which is like a lot of congestion on the transmission system [27, 28, 29]. Thus, we can consider that DLMP is the additional cost of transferring one more Megawatt (MW) at any feeder.

In a transmission system, if there is no any congestion and losses, all LMPs would be the same called Market Clearing Price (MCP) because it reflects only the cost of serving the next increment of the load, otherwise, the bus price will differ. Generally, LMPs are different among locations due to transmission losses and constraints for the next cheapest MW of electric energy can reach all locations in this system. Therefore, when the next cheapest MW can reach all locations, the cost of physical transmission losses will result in different LMPs across this system. Obviously, to understand cost of energy, a congestion charge, and transmission system losses are the three most important components of LMPs calculation.

In addition, day-ahead and real-time LMPs and DLMPs are two different types of methods for calculating prices in a power system. However, they are based on the same basic calculation using the different nodal prices, optimizing the dispatches and minimizing the costs for energy, congestion, and losses [30, 31]. 


\section{Chapter 3}

\section{Entities Modeling Layer}

In the entities modeling layer, we simulate the generation, transmission, distribution and consumption of modified IEEE 13 node test feeder system. Moreover, we consider two MGs and three PV panels as generators in this power system topology. On the other hand, smart batteries are playing vital roles in the management and integration of power systems with renewable energy resources [32]. Therefore, the smart net meters would monitor and communicate with the Energy Management System (EMS). They are the two most different components between entities modeling layer and previous DERs layer because they would be programmed by smart contracts of blockchain on Ethereum for managing and delivering power and energy exactly.

For the modified IEEE 13 node test feedr system topology [33], parameters of generators, transmission or feeder lines, and loads are key to solve the Optimal Power Flow (OPF) and DLMPs problems. As a result, this chapter is about data sets. To begin with, it is better to analyze any power system by drawing its topology. Figure 3.1 demonstrates the topology of the modified IEEE 13 node test feeder system. It can be noticed that there are several generators on nodes $650,646,633,684,692$, and 680 respectively. Node 650 is connected to the main grid; node 646, 633, and 
684 are the solar PV generators; node 692 and 680 are microgrids. The generation cost parameters and limitation data are shown in Table 3.1. Load are on nodes of $646,645,632,634,611,671,675$, and 652 proportionally. The load data listed in Table 3.2. And, Table 3.3 and Table 3.4 display line segment parameters are of IEEE 13 node test feeder system including line impedances, connections and length $[33]$.

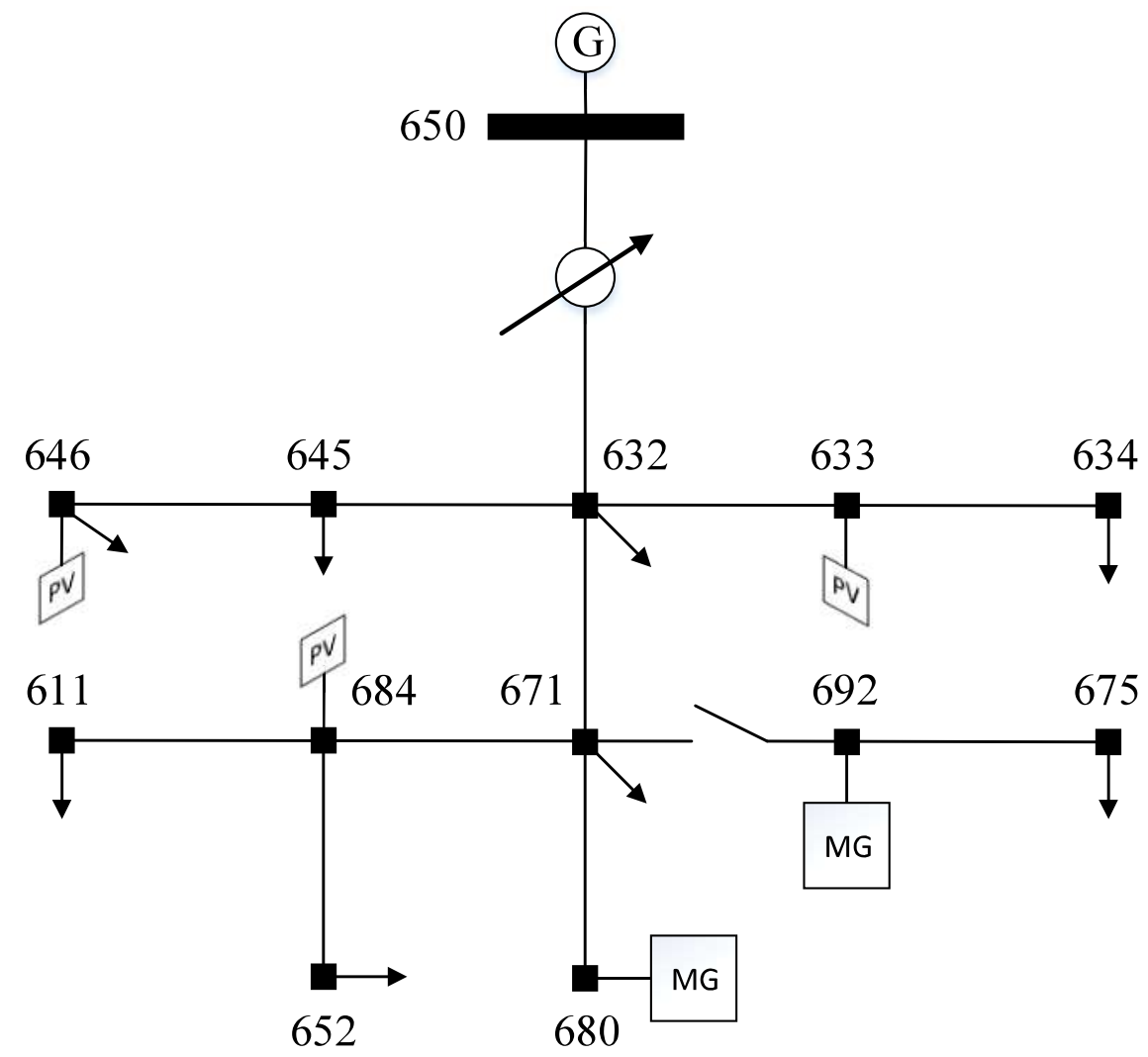

Figure 3.1: Modified IEEE 13 node test feeder system topology 
Table 3.1: Generator parameters

\begin{tabular}{|c|c|c|c|c|}
\hline Node & $a_{n}\left(\$ / p u^{2}\right)$ & $b_{n}(\$ / p u)$ & $c_{n}$ & $\overline{P_{n}}(\mathrm{~kW})$ \\
\hline Sourcebus.1 & 0.36 & 20.7 & 0 & 1100 \\
\hline 646.3 & 0.056 & 3.5 & 0 & 185 \\
\hline 633.1 & 0.07 & 4.0 & 0 & 185 \\
\hline 684.1 & 0.058 & 3.5 & 0 & 185 \\
\hline 692.1 & 0.082 & 4.5 & 0 & 600 \\
\hline 692.2 & 0.082 & 4.5 & 0 & 600 \\
\hline 692.3 & 0.082 & 4.5 & 0 & 600 \\
\hline 680.1 & 0.068 & 3.0 & 0 & 550 \\
\hline 680.2 & 0.068 & 3.0 & 0 & 550 \\
\hline 680.3 & 0.068 & 3.0 & 0 & 550 \\
\hline
\end{tabular}

Table 3.2: Load parameters

\begin{tabular}{|c|c|c|c|c|}
\hline Node & $a_{n}\left(\$ / p u^{2}\right)$ & $b_{n}(\$ / p u)$ & $c_{n}$ & $\overline{P_{n}}(\mathrm{~kW})$ \\
\hline 646.2 & 0.084 & 8.0 & 0 & -230 \\
\hline 645.2 & 0.074 & 7.0 & 0 & -170 \\
\hline 632.1 & 0.068 & 6.4 & 0 & 0 \\
\hline 634.1 & 0.08 & 7.5 & 0 & -160 \\
\hline 634.2 & 0.06 & 6.3 & 0 & -120 \\
\hline 634.3 & 0.07 & 8.0 & 0 & -90 \\
\hline 611.3 & 0.076 & 7.0 & 0 & -80 \\
\hline 671.1 & 0.07 & 7.5 & 0 & -385 \\
\hline 671.2 & 0.08 & 8.0 & 0 & -385 \\
\hline 671.3 & 0.07 & 7.2 & 0 & -220 \\
\hline 675.1 & 0.064 & 6.8 & 0 & -485 \\
\hline 675.2 & 0.04 & 7.5 & 0 & -68 \\
\hline 675.3 & 0.06 & 8.0 & 0 & -212 \\
\hline 652.1 & 0.065 & 6.9 & 0 & -128 \\
\hline
\end{tabular}


Table 3.3: Line parameters

\begin{tabular}{|c|c|c|c|c|}
\hline Node A & Node B & Length (ft.) & Phasing & Impedances Config. \\
\hline 632 & 645 & 500 & C B N & 603 \\
\hline 635 & 633 & 500 & C A B N & 602 \\
\hline 645 & 646 & 300 & C B N & 603 \\
\hline 650 & 632 & 2000 & B A C N & 601 \\
\hline 684 & 652 & 800 & A N & 607 \\
\hline 632 & 671 & 2000 & B A C N & 601 \\
\hline 671 & 684 & 300 & A C N & 604 \\
\hline 671 & 680 & 1000 & B A C N & 601 \\
\hline 684 & 611 & 300 & C N & 605 \\
\hline 692 & 675 & 500 & A B C N & 606 \\
\hline
\end{tabular}

Table 3.4: Line impedances configuration table

\begin{tabular}{|c|c|c|c|c|c|c|c|c|}
\hline \multirow{2}{*}{ Config. } & \multicolumn{2}{|c|}{601} & \multicolumn{2}{|c|}{602} & \multicolumn{2}{|c|}{603} & \multicolumn{2}{|c|}{604} \\
\hline & Res. & Rea. & Res. & Rea. & Res. & Rea. & Res. & Rea. \\
\hline$A-B$ & 0.1560 & 0.5017 & 0.1580 & 0.4236 & 0.0 & 0.0 & 0.0 & 0.0 \\
\hline$A-C$ & 0.1580 & 0.4236 & 0.1560 & 0.5017 & 0.0 & 0.0 & 0.2066 & 0.4591 \\
\hline $\mathrm{A}-\mathrm{N}$ & 0.3465 & 1.0179 & 0.7526 & 1.1814 & 0.0 & 0.0 & 1.3238 & 1.3569 \\
\hline $\mathrm{B}-\mathrm{C}$ & 0.1535 & 0.3849 & 0.1535 & 0.3849 & 0.2066 & 0.4591 & 0.0 & 0.0 \\
\hline $\mathrm{B}-\mathrm{N}$ & 0.3375 & 1.0478 & 0.7475 & 1.1983 & 1.3294 & 1.3471 & 0.0 & 0.0 \\
\hline $\mathrm{C}-\mathrm{N}$ & 0.3414 & 1.0348 & 0.7436 & 1.2112 & 1.3238 & 1.3569 & 1.3294 & 1.3471 \\
\hline \multirow{2}{*}{ Config. } & \multicolumn{2}{|c|}{605} & \multicolumn{2}{|c|}{606} & \multicolumn{2}{|c|}{607} & & \\
\hline & Res. & Rea. & Res. & Rea. & Res. & Rea. & & \\
\hline$A-B$ & 0.0 & 0.0 & 0.3192 & 0.0328 & 0.0 & 0.0 & & \\
\hline $\mathrm{A}-\mathrm{C}$ & 0.0 & 0.0 & 0.2849 & -0.0143 & 0.0 & 0.0 & & \\
\hline $\mathrm{A}-\mathrm{N}$ & 0.0 & 0.0 & 0.7982 & 0.4463 & 1.3425 & 0.5124 & & \\
\hline $\mathrm{B}-\mathrm{C}$ & 0.0 & 0.0 & 0.3192 & 0.0328 & 0.0 & 0.0 & & \\
\hline $\mathrm{B}-\mathrm{N}$ & 0.0 & 0.0 & 0.7891 & 0.4041 & 0.0 & 0.0 & & \\
\hline $\mathrm{C}-\mathrm{N}$ & 1.3292 & 1.3475 & 0.7982 & 0.4463 & 0.0 & 0.0 & & \\
\hline
\end{tabular}


For the p.u. calculation of power system, we consider the base apparent power $S_{\text {base }}=100 \mathrm{KVA}$, and base voltage $V_{\text {base }}=115 \mathrm{kV}$. And then, use formulas (3.1.1), (3.1.2), (3.1.3) and (3.1.4) to calculate the current, impedance, admittance and voltage with the base values.

$$
\begin{gathered}
I_{\text {base }}=\frac{S_{\text {base }}}{V_{\text {base }} \times \sqrt{3}} \\
Z_{\text {base }}=\frac{V_{\text {base }}}{I_{\text {base }} \times \sqrt{3}} \times \text { Length } \\
Y_{\text {base }}=\frac{1}{Z_{\text {base }}} \times \text { Length }
\end{gathered}
$$

In addition to generator, load and line parameters, the load input values are important to know also. Figure 3.2 shows the a day load input profiles in $\mathrm{kW}$. In this thesis, all OPF problems are solved by distributed consensus + innovations approach. The detailed explanation of this algorithm is in Chapter 4.

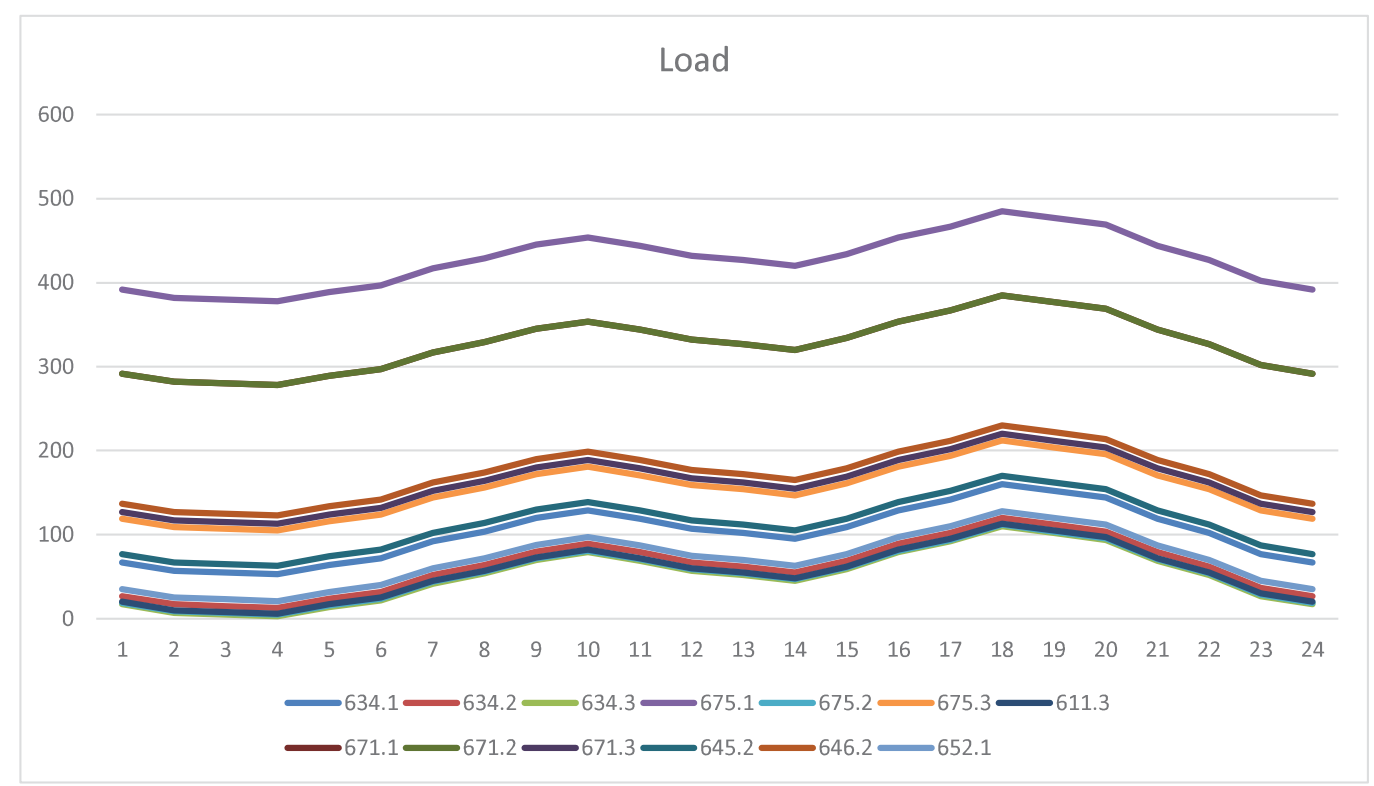

Figure 3.2: Load input profile 


\section{Chapter 4}

\section{Price Mechanism Layer}

In order to complete this distributed price mechanism layer, the consensus + innovations approach for distributed multi-agent coordination is introduced in this chapter [34]. The main idea of this approach is to optimize the global value according to local and neighborhood information autonomously. In general, the updated item of each iteration of $\lambda$ and $P$ in different time scale are given by (4.0.1) and (4.0.2). Equation (4.0.1) shows that 1 ) there are $i$ iterations, $j$ nodes and $t$ hours; 2) $\alpha_{i}$ and $\beta_{i}$ are tuning parameters to 0 when iteration goes to infinity; 3) $\omega_{j}$ is a set of communication neighborhood which comes from physical entity modeling topology connection. It is a projection operation in equation (4.0.2) for power in-

jected/consumed $P_{j, t}^{i+1}$ onto the interval $\left[P_{n, t}^{\min }, P_{n, t}^{\max }\right]$ using new $\lambda_{j, t}^{i+1}$ value and generator cost parameter $a_{n}$ and $b_{n}$. The updated iteration flow chart of the consensus + innovations is demonstrated in Figure 4.1.

$$
\begin{aligned}
& \lambda_{j, t}^{i+1}=\lambda_{j, t}^{i}-\beta_{i} \sum_{l \in \omega_{j}}\left(\lambda_{j, t}^{i}-\lambda_{l, t}^{i}\right)-\alpha_{i} \sum_{n \in \Omega_{j}} P_{n, t}^{i} \\
& P_{j, t}^{i+1}=\underset{P_{n, t}^{\text {min }} \leq P_{n, t} \leq P_{n, t}^{\max }}{\arg }\left\|P_{n, t}^{i}-\frac{\lambda_{j, t}^{i+1}-b_{n, t}}{a_{n, t}}\right\|^{2}
\end{aligned}
$$




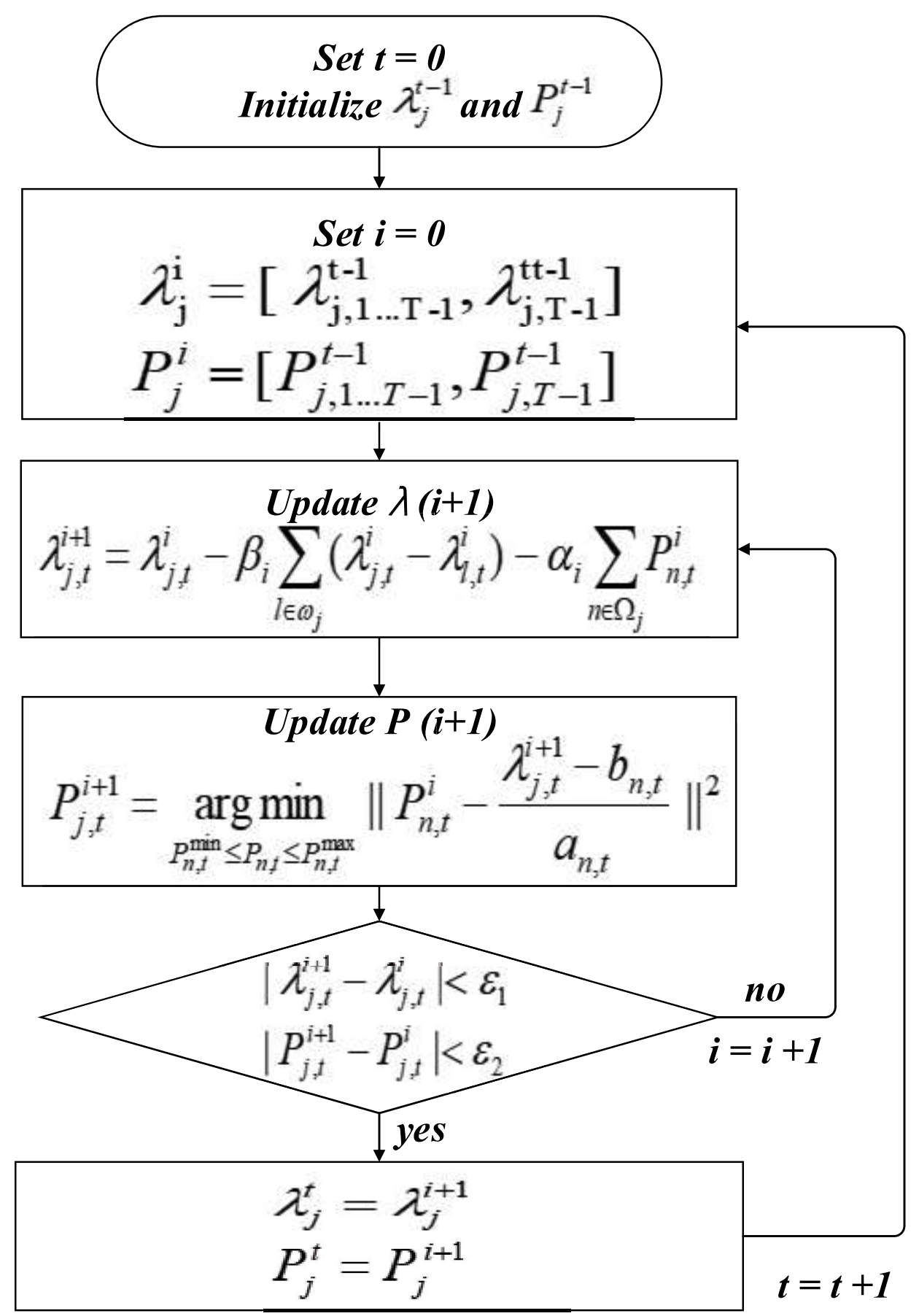

Figure 4.1: Consensus + innovations approach flow chart 
In our DCOPF case $[35,36,37]$, the quadratic cost function is used to model the generation costs for each generator $n$ from Figure 3.1 given by:

$$
C_{n}\left(P_{n}\right)=\frac{1}{2} a_{n} P_{n}^{2}+b_{n} P_{n}+c_{n}
$$

Where $a_{n}, b_{n}, c_{n} \geq 0$

In this layer, the goal is to determine the DLMPs for each node which minimizes the total system cost. The marginal cost function is given by:

$$
\frac{d C_{n}\left(P_{n}\right)}{d P_{n}}=a_{n} P_{n}+b_{n}=\lambda_{n}
$$

Where $P_{n}$ is limited to $\underline{P}_{n}$ and $\bar{P}_{n}$, when $\bar{P}_{n} \geq 0$, it is for generator, when $\bar{P}_{n} \geq 0$, it is for load, and when $\underline{P}_{n} \leq 0, \bar{P}_{n} \geq 0$ it is for storage or battery

The mathematical problem formulation of generation costs modeling can be expressed by:

$$
\begin{gathered}
\text { obj. } \min _{P_{n}} \sum_{n \in \Omega_{G}}\left(C_{n}\left(P_{n}\right)\right)=\min _{P_{n}} \sum_{n \in \Omega_{G}}\left(\frac{1}{2} a_{n} P_{n}^{2}+b_{n} P_{n}+c_{n}\right) \\
\qquad \underline{P}_{n} \leq P_{n} \leq \bar{P}_{n} \\
\text { s.t. } \quad \forall n \in \Omega_{G} \\
\sum_{n \in \Omega_{G}} P_{n}-P_{L_{o a d}}=\sum_{j \in \Omega_{k}} \frac{\theta_{k}-\theta_{j}}{X_{k j}} \quad \forall n \in \Omega_{N} \\
-\bar{P}_{k j} \leq \frac{\theta_{k}-\theta_{j}}{X_{k j}} \leq \bar{P}_{k j} \quad \forall k j \in \Omega_{L}
\end{gathered}
$$$$
\theta_{1}=0
$$ 
Where: $P_{n}$ :

$$
\begin{aligned}
& a_{n}, b_{n}, c_{n}: \\
& P_{\text {Load }_{k}}: \\
& \theta_{k}: \\
& X_{k j}: \\
& \underline{P}_{n}, \bar{P}_{n}: \\
& -\bar{P}_{k j}, \bar{P}_{k j}: \\
& \Omega_{k}: \\
& \Omega_{N}: \\
& \Omega_{G}: \\
& \Omega_{L}:
\end{aligned}
$$

generation at generator $n$

quadratic cost parameters of generator $n$

load at bus $\mathrm{k}$

angle at bus $\mathrm{k}$

reactance of line segment between bus $k$ and $j$

generation minimum, maximum at generator $\mathrm{n}$

line capacity between bus $\mathrm{k}$ and $\mathrm{j}$

set of all bus connected to bus $\mathrm{k}$

set of all nodes

set of all generators

set of all line segments

The Lagrange function for this generation cost minimizing problem is given by:

$$
\begin{aligned}
\mathcal{L}= & \sum_{n \in \Omega_{G}}\left(\frac{1}{2} a_{n} P_{n}^{2}+b_{n} P_{n}+c_{n}\right)+ \\
& \sum_{n \in \Omega_{G}} \mu_{n}^{+}\left(P_{n}-\bar{P}_{n}\right)+\sum_{n \in \Omega_{G}} \mu_{n}^{-}\left(-P_{n}-\underline{P}_{n}\right)+ \\
& \sum_{i=1}^{\Omega_{N}} \lambda_{i}\left(-\sum_{n \in \Omega_{G}} P_{n}+P_{\text {Load }_{k}}+\sum_{j \in \Omega_{k}} \frac{\theta_{k}-\theta_{j}}{X_{k j}}\right)+ \\
& \sum_{k j \in \Omega_{L}} \mu_{k j}\left(\frac{\theta_{k}-\theta_{j}}{X_{k j}}-\bar{P}_{k j}\right)+\sum_{k j \in \Omega_{L}} \mu_{k j}\left(-\frac{\theta_{k}-\theta_{j}}{X_{k j}}-\bar{P}_{k j}\right)+\lambda_{1} \theta_{1}
\end{aligned}
$$

where $\lambda$ and $\mu$ are Lagrange multipliers. The first order optimality conditions are given by:

$$
\frac{d \mathcal{L}}{d P_{n}}=a_{n} P_{n}+b_{n}+\mu_{n}^{+}-\mu_{n}^{-}-\lambda_{n}=0
$$




$$
\begin{aligned}
& \frac{d \mathcal{L}}{d \lambda_{k}}=-\sum_{n \in \Omega_{G}} P_{n}+P_{\text {Load }_{k}}+\sum_{j \in \Omega_{k}} \frac{\theta_{k}-\theta_{j}}{X_{k j}}=0 \\
& \frac{d \mathcal{L}}{d \theta_{k}}=\lambda_{k} \sum_{j \in \Omega_{k}} \frac{1}{X_{k j}}-\sum_{j \in \Omega_{k}} \lambda_{k} \frac{1}{X_{k j}}+\sum_{j \in \Omega_{k}}\left(\mu_{k j}-\mu_{j k}\right) \frac{1}{X_{k j}}=0 \\
& \frac{d \mathcal{L}}{d \lambda_{1}}=\theta_{1}=0 \\
& \frac{d \mathcal{L}}{d \mu_{n}^{+}}=P_{n}-\bar{P}_{n} \leq 0 \\
& \frac{d \mathcal{L}}{d \mu_{n}^{-}}=-P_{n}-\underline{P}_{n} \leq 0 \\
& \frac{d \mathcal{L}}{d \mu_{k j}}=\frac{\theta_{k}-\theta_{j}}{X_{k j}}-\bar{P}_{k j} \leq 0 \\
& \frac{d \mathcal{L}}{d \mu_{k j}}=-\frac{\theta_{k}-\theta_{j}}{X_{k j}}-\bar{P}_{k j} \leq 0
\end{aligned}
$$

Now, apply our DCOPF case into equation (4.0.1) and (4.0.2) of distributed consensus + innovations approach. Thus, the updated Lagrange multipliers $\lambda_{j, t}^{i+1}$, $P_{j, t}^{i+1}, \theta_{j, t}^{i+1}, \mu_{i j, t}^{i+1}$ and $\mu_{j i, t}^{i+1}$ are given by:

$$
\begin{aligned}
\lambda_{j, t}^{i+1}=\lambda_{j, t}^{i} & -\beta_{i}\left(\lambda_{j, t}^{i} \sum_{j \in \Omega_{k}} \frac{1}{X_{k j}}-\sum_{j \in \Omega_{k}} \lambda_{j, t}^{i} \frac{1}{X_{k j}}+\sum_{j \in \Omega_{k}}\left(\mu_{k j, t}-\mu_{j k, t}\right) \frac{1}{X_{k j}}\right) \\
& -\alpha_{i}\left(\sum_{n \in \Omega_{G}} P_{n, t}^{i}-P_{\text {Load }}-\sum_{j \in \Omega_{k}} \frac{\theta_{k, t}^{i}-\theta_{j, t}^{i}}{X_{k j}}\right)
\end{aligned}
$$




$$
\begin{gathered}
P_{n, t}^{i+1}=\underset{P_{n, t}^{m i n} \leq P_{n, t} \leq P_{n, t}^{\max }}{\boldsymbol{a r g} \boldsymbol{\operatorname { m i n }}}\left\|P_{n, t}^{i}-\frac{\lambda_{j, t}^{i+1}-b_{n, t}}{a_{n, t}}\right\|^{2} \\
\theta_{j, t}^{i+1}=\theta_{j, t}^{i}-\gamma\left(-\sum_{n \in \Omega_{G}} P_{n, t}^{i+1}+P_{\text {Load }_{k}}+\sum_{j \in \Omega_{k}} \frac{\theta_{k, t}^{i}-\theta_{j, t}^{i}}{X_{k j}}\right) \\
\mu_{k j, t}^{i+1}=\underset{\mu_{k j, t}^{\text {min }} \leq \mu_{k j, t} \leq \mu_{k j, t}^{\max }}{\boldsymbol{a r g}}\left\|\mu_{k j, t}^{i}-\delta\left(\bar{P}_{k j}-\frac{\theta_{k, t}^{i+1}-\theta_{j, t}^{i+1}}{X_{k j}}\right)\right\|^{2} \\
\mu_{j k, t}^{i+1}=\underset{\mu_{j k, t}^{\min } \leq \mu_{j k, t} \leq \mu_{j k, t}^{\max }}{\boldsymbol{\operatorname { a r g }}}\left\|\mu_{j k, t}^{i}-\delta\left(\bar{P}_{j k}-\frac{\theta_{k, t}^{i+1}-\theta_{j, t}^{i+1}}{X_{k j}}\right)\right\|^{2}
\end{gathered}
$$

1) $\bar{P}_{i j}$ and $\bar{P}_{j i}$ are the line limits between feeder $i$ and $j$. They are $500 \mathrm{~kW}$;

2) $\Omega_{i}$ is a set of communication neighborhood which comes from physical entity modeling topology connection;

3) $\Omega_{N}, \Omega_{G}$, and $\Omega_{L}$ are set of nodes, generators and line segments;

4) using new updated value for next step calculation such as $\lambda_{j, t}^{i+1}$ in (4.0.19), $P_{n, t}^{i+1}$ in (4.0.20) and $\theta_{j, t}^{i+1}$ and $\mu_{j, t}^{j+1}$ in (4.0.21), (4.0.22) and (4.0.23);

5) $\alpha, \beta, \gamma$, and, $\delta$ are the four key tunning parameters to impact the convergence. They are set to:

$$
\begin{array}{ll}
\alpha=\frac{0.55}{i^{0.98}} & \beta=\frac{0.2}{i^{0.001}} \\
\gamma=\frac{0.05}{i^{0.001}} & \delta=\frac{0.008}{i^{0.001}}
\end{array}
$$

where $i$ is the iteration number;

The application of DCOPF into the consensus + innovations approach flow chart is show in Figure 4.2. The updated terms $\lambda_{j, t}^{i+1}, P_{n, t}^{i+1}, \theta_{j, t}^{i+1}, \mu_{i j, t}^{i+1}$ and $\mu_{j i, t}^{i+1}$ are accorded to equations (4.0.19) - (4.0.23).

We simulated 24 hours $(\mathrm{t}=24)$ for two cases. The generation units output of the two cases are shown in Figure 4.3 and 4.4. In Figure 4.4, the generation output of three PVs are 0 at hour 9 and 10, and the generation of the generator 


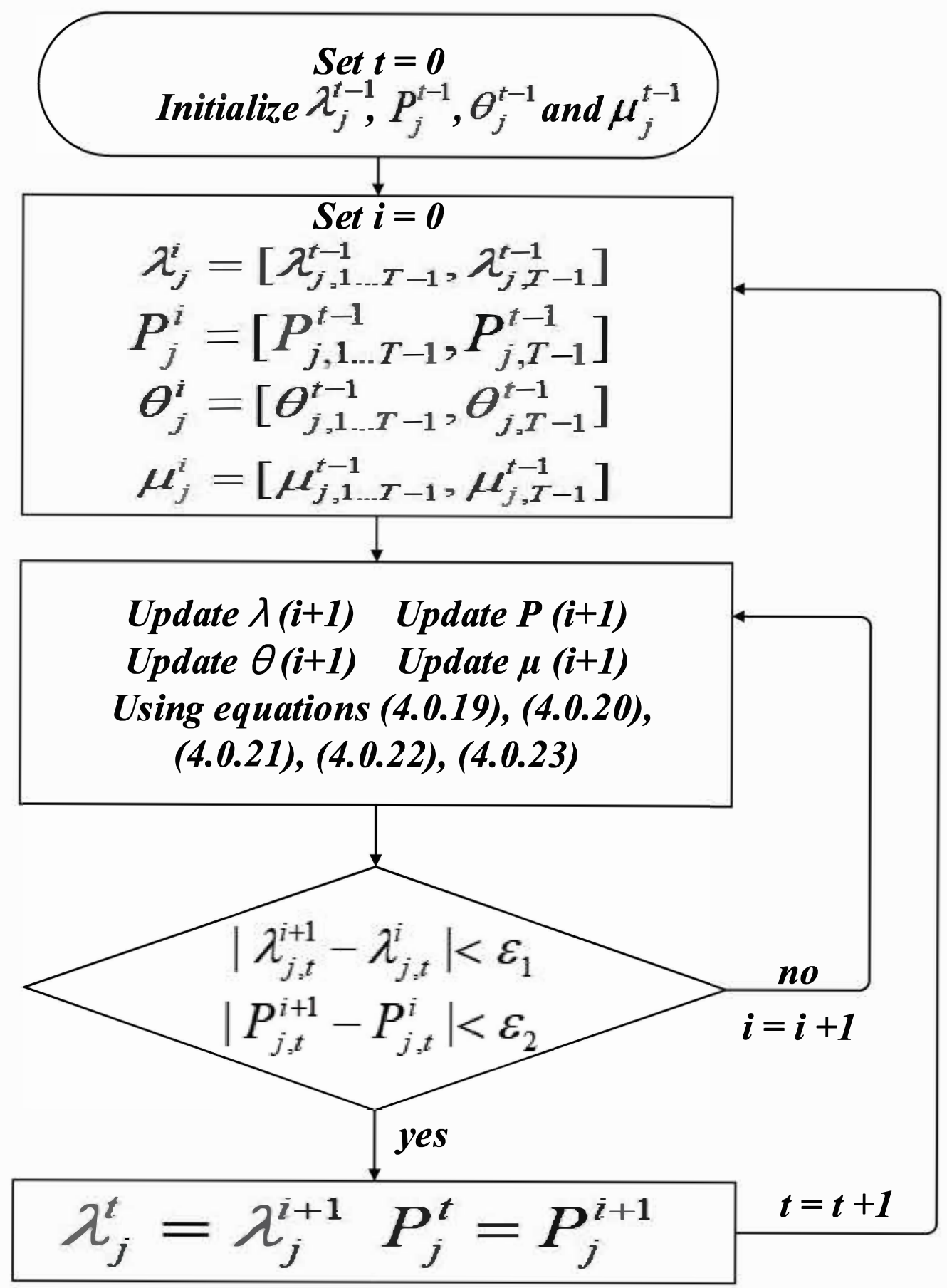

Figure 4.2: DCOPF consensus + innovations approach flow chart 
which is connected to the main grid is 0 at hour 14 and 15. Figure 4.5 and 4.6 are the distributed DLMPs for each unit. It can be observed that the prices increase when PVs generation is low such as at hours 9 and 10. In addition, Figure 4.7 and 4.8 show the 1000th iteration of distributed DLMPs and generation output in the normal case at bus 1, 10, 14 and 18; while Figure 4.9 and 4.10 demonstrate the 1000th iteration of distributed DLMPs and generation output in the congested case.

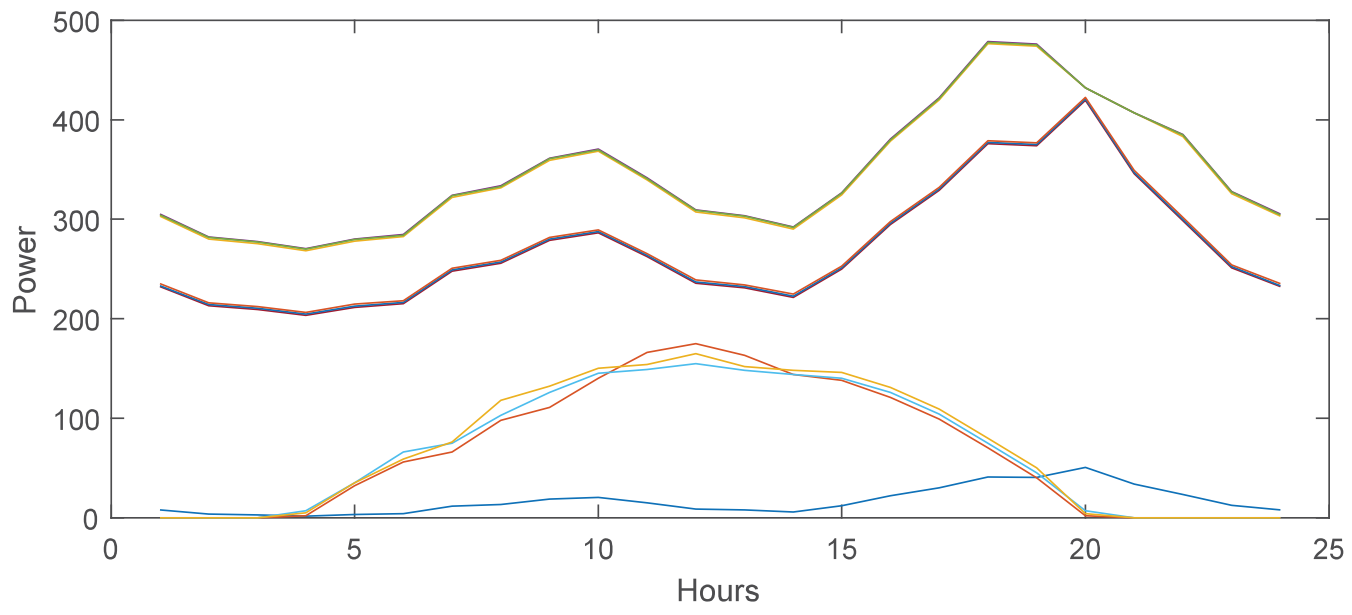

Figure 4.3: Generation units output

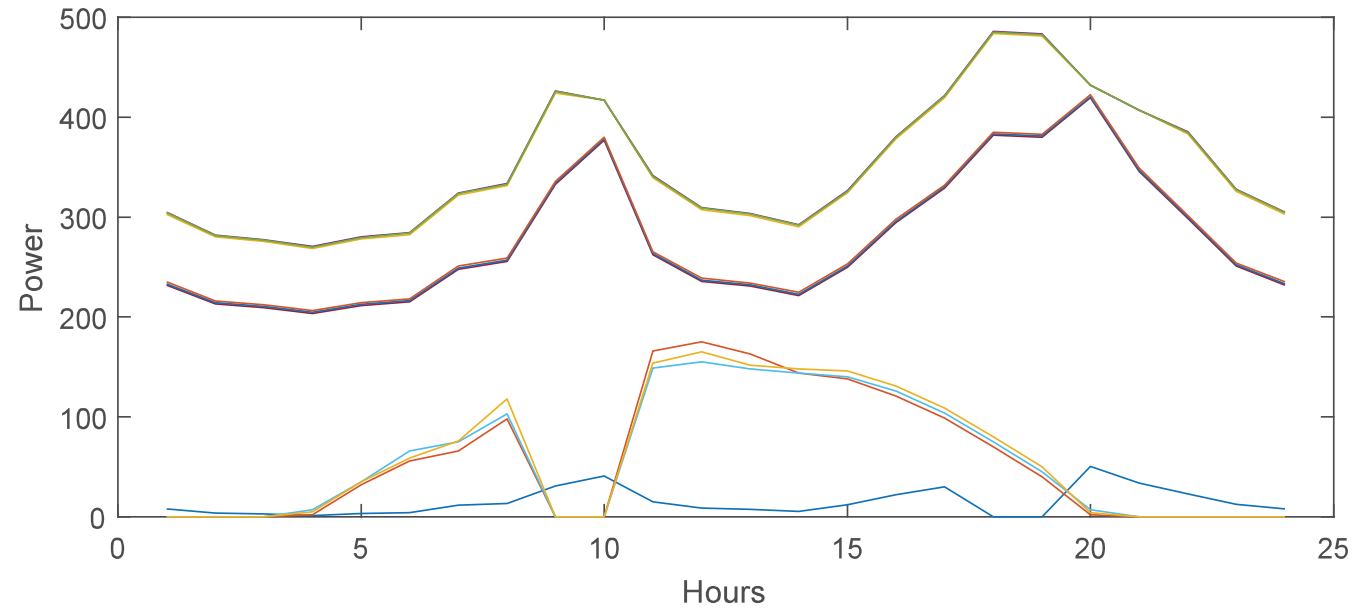

Figure 4.4: Generation units output for congested case 


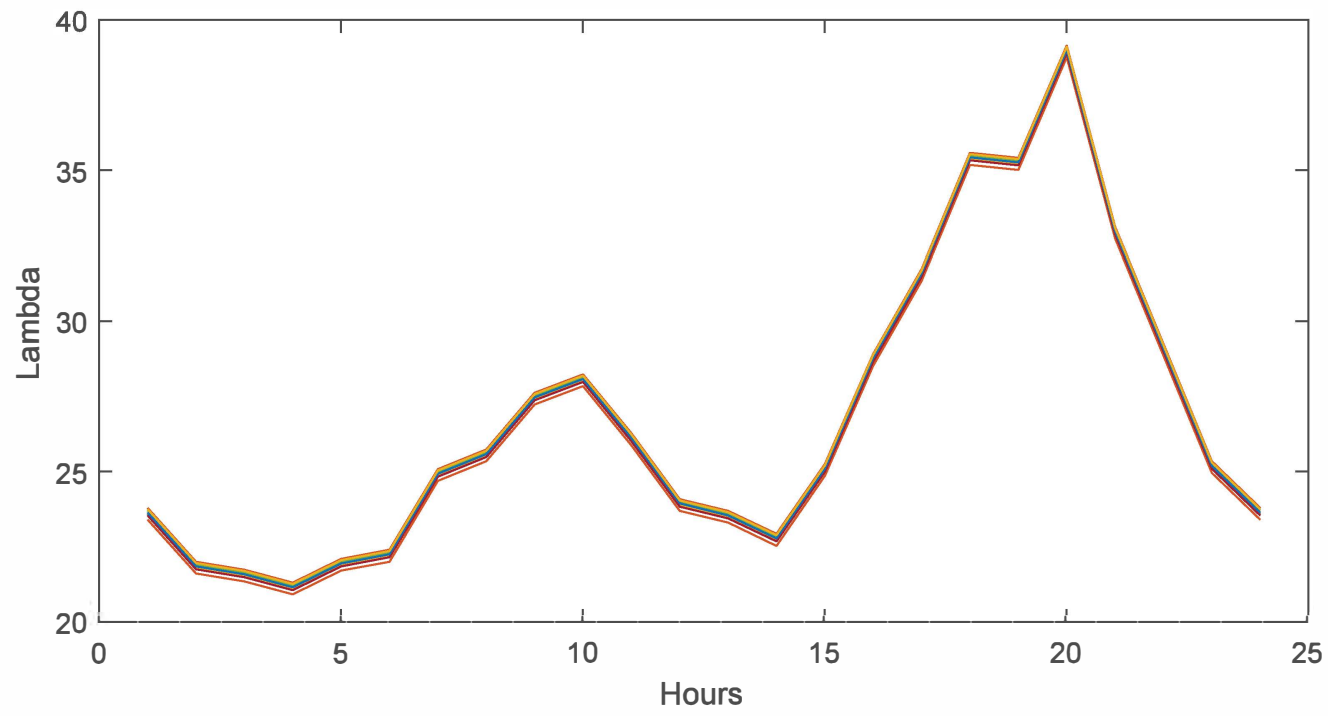

Figure 4.5: Distributed DLMPs

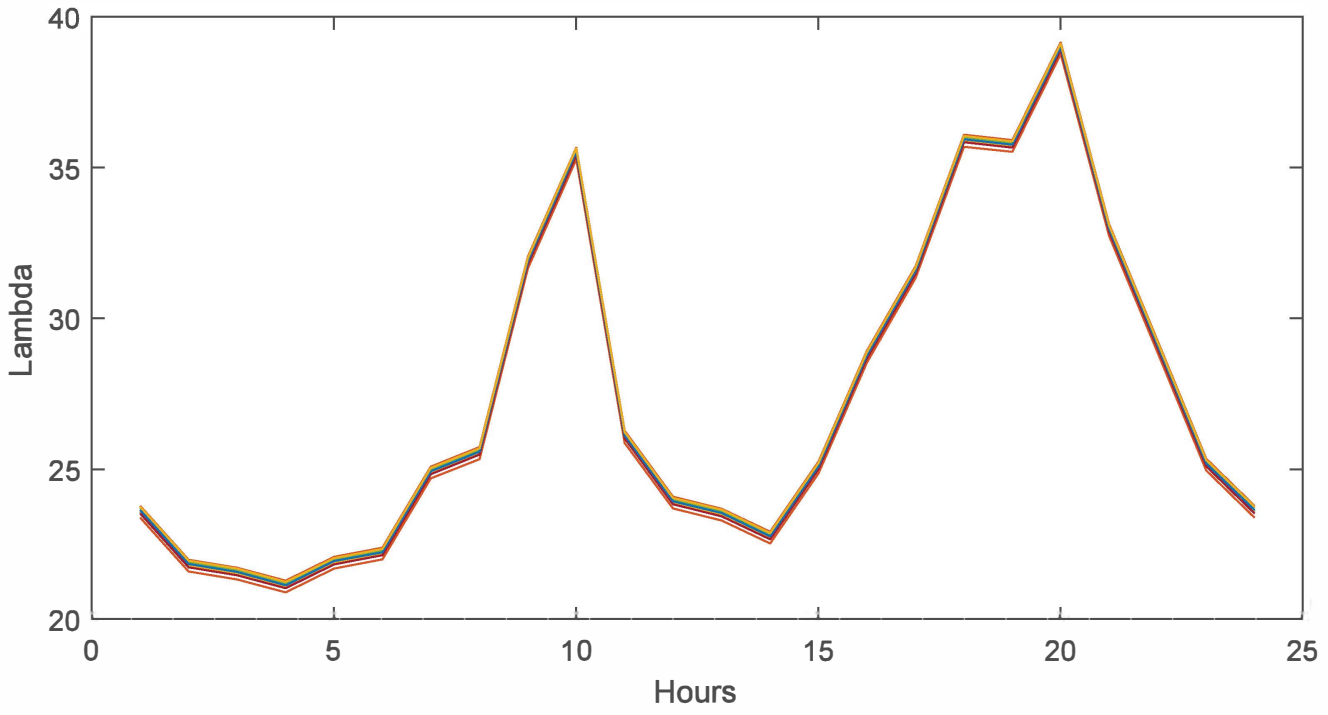

Figure 4.6: Distributed DLMPs for congested case 

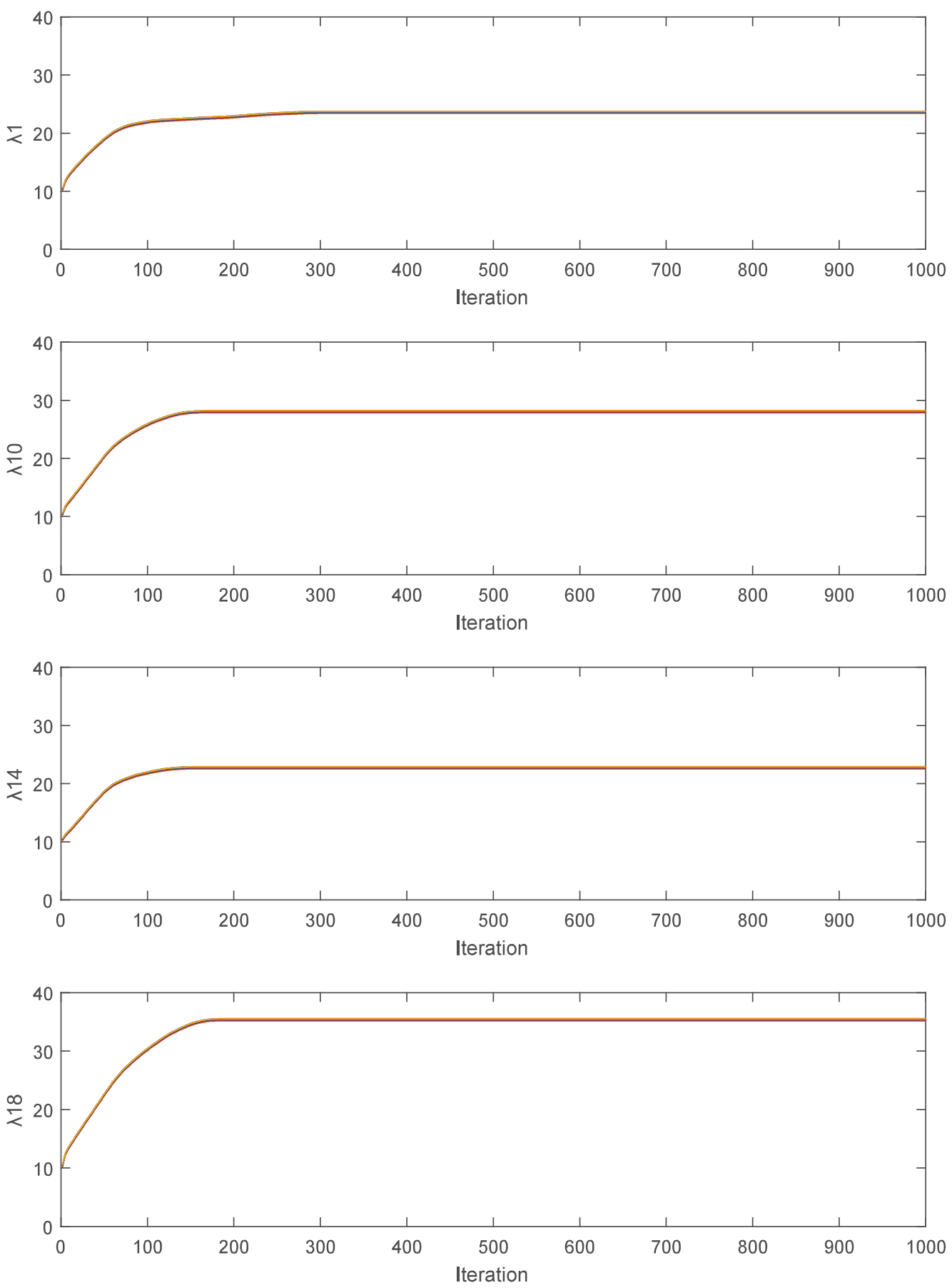

Figure 4.7: The 1000th iteration of distributed DLMPs at hour 1, 10, 14, and 18 

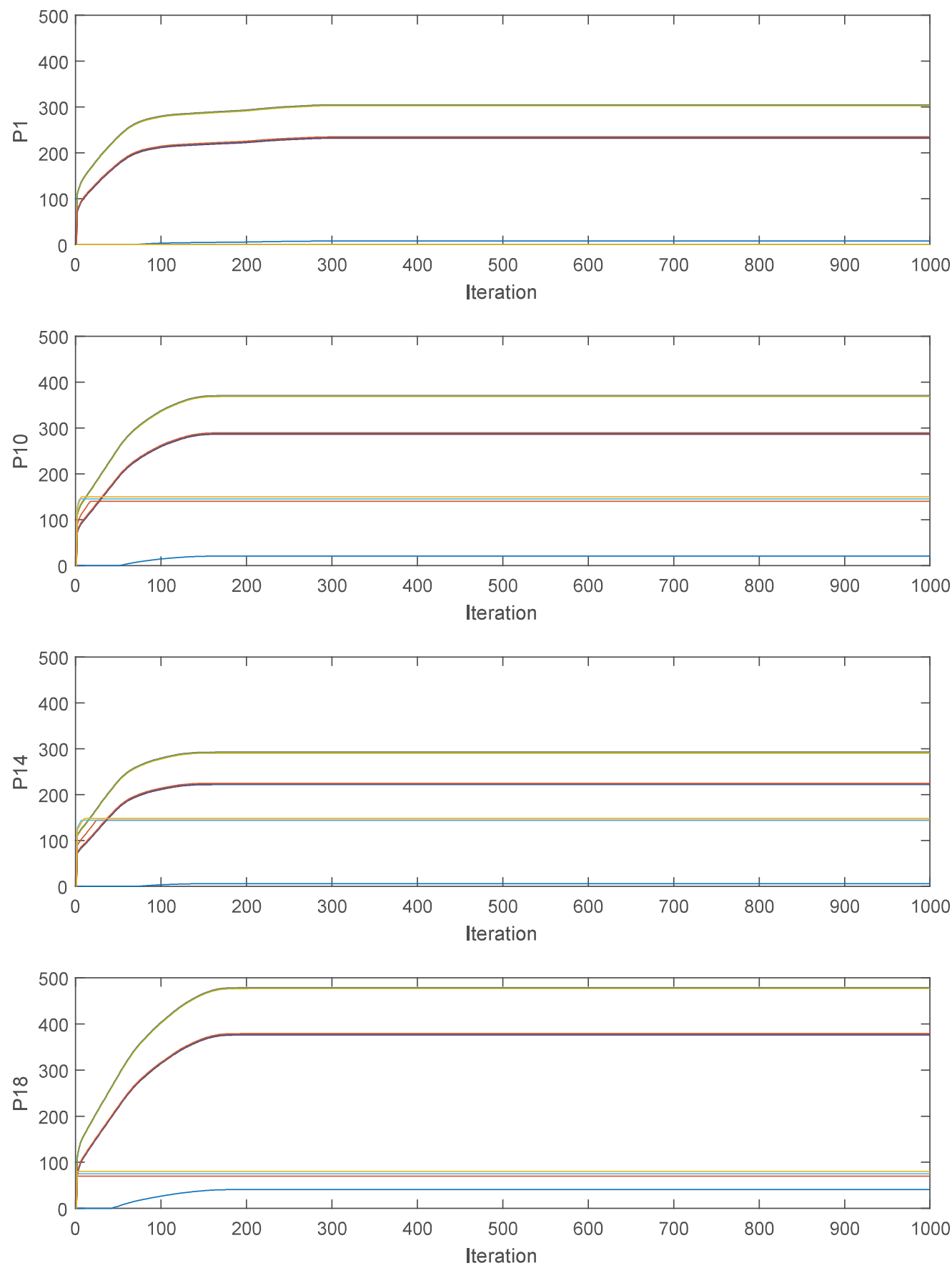

Figure 4.8: The 1000th iteration of generation output at hour 1, 10, 14, and 18 

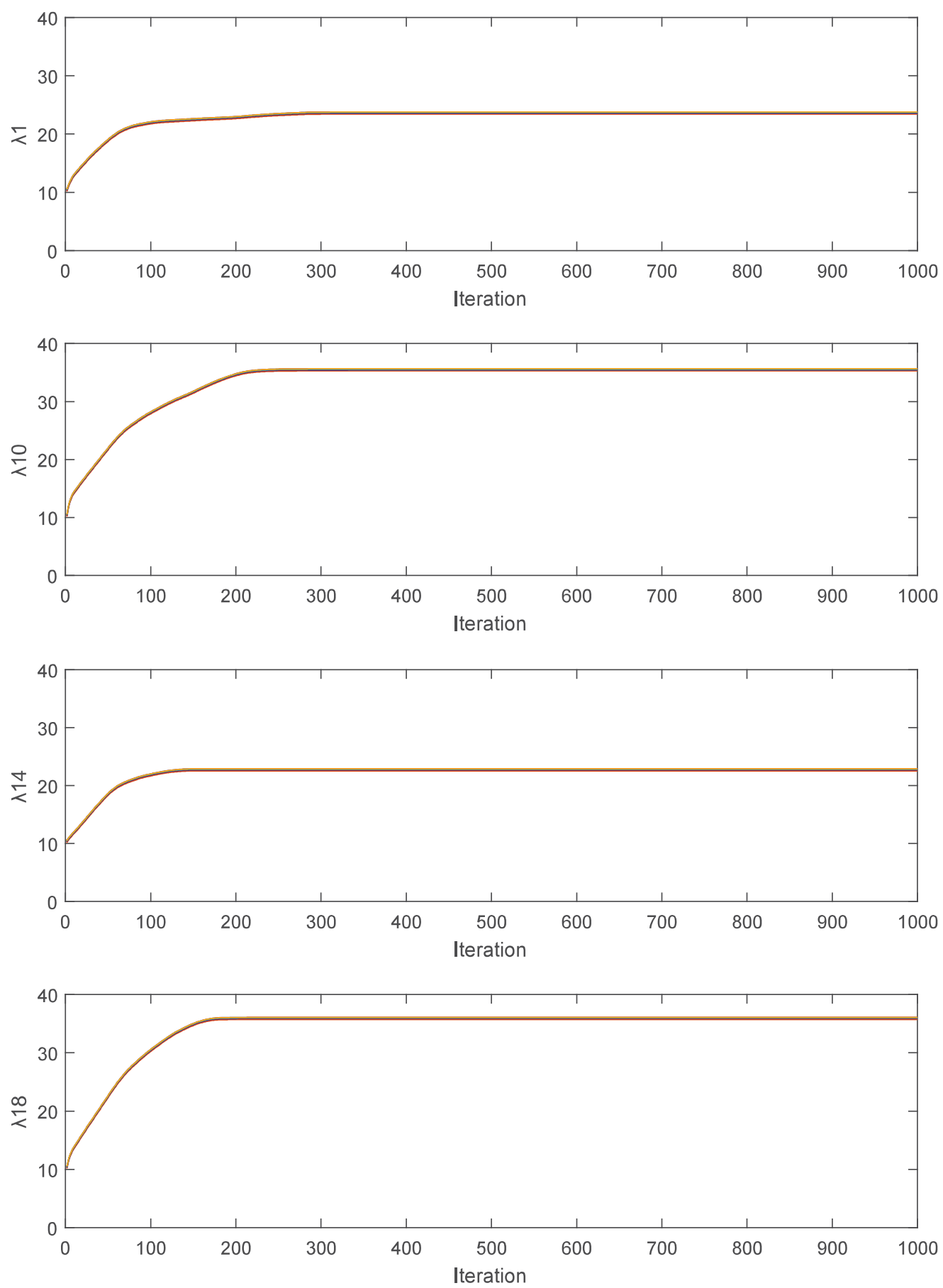

Figure 4.9: The 1000th iteration of distributed DLMPs in the congested case 

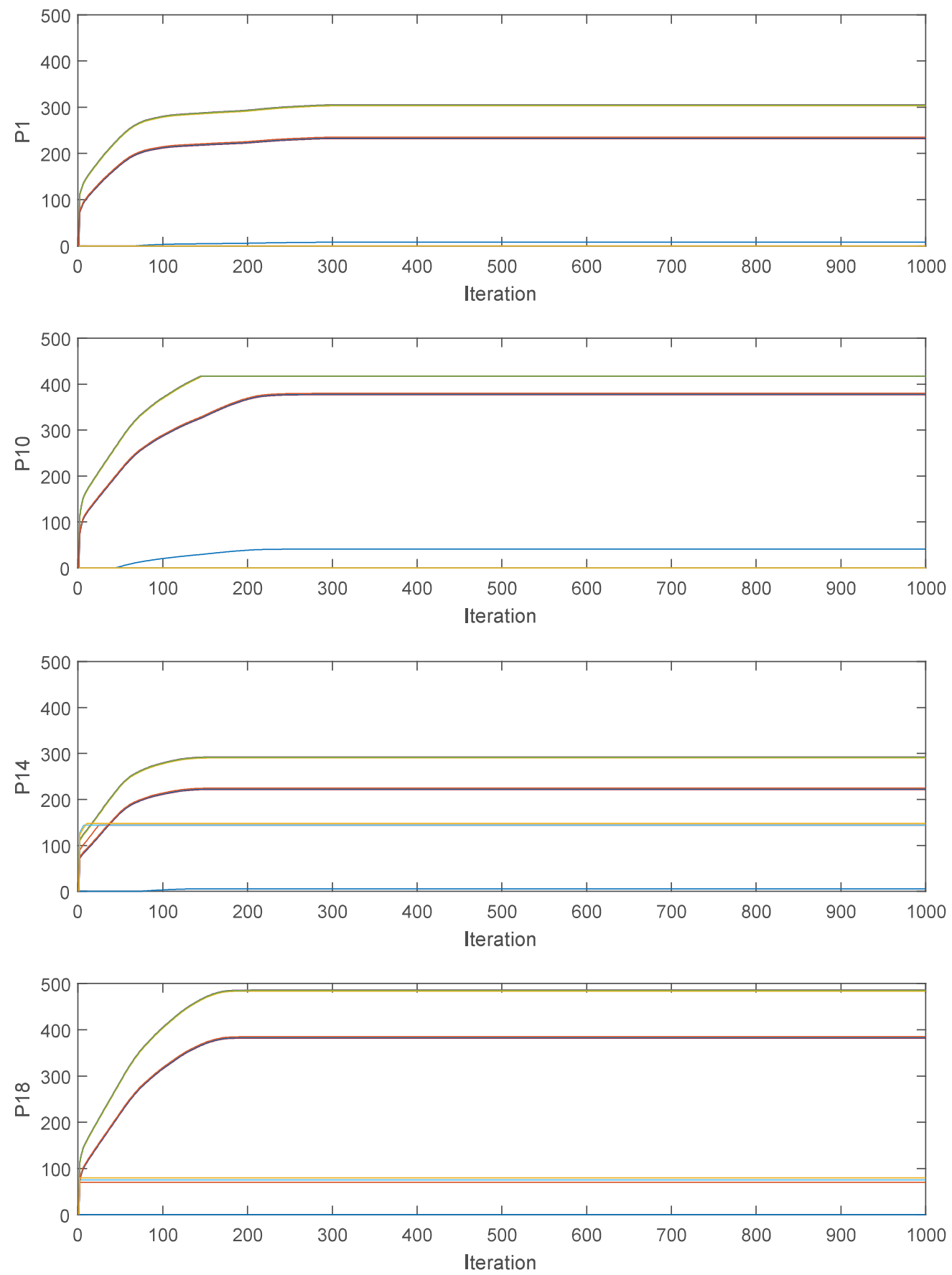

Figure 4.10: The 1000th iteration of generation output in the congested case 


\section{Chapter 5}

\section{PILT-DAO Market Layer}

We will consider connecting all components in this PILT-DAO market layer, after setting entities modeling and price mechanism layers. In the third layer, DAO will be used to design the decentralized microgrid market layer on Ethereum private blockchain with the parallel methodology. So, there are two systems instantaneously such as real and artificial ITL-DAO system by combining a PILT-DAO market using ACP method.

\subsection{Whole PILT-DAO Blueprint}

In this section, the whole PILT-DAO layers blueprint is demonstrated in Figure 5.1 showing how all elements in the three layers are connected together.

According to Figure 5.1, The three layers are briefly summarized. Firstly, there are lots of facilities in entities modeling layer such as PV solar panels, microgrids, smart batteries and smart net meters for generating, transmitting, distributing, and consuming. We number those assets in Figure 5.1, for example, microgrid\#1 contains smart battery\#1 and smart net meter\#1, and PV solar panel\#1 is connected to smart battery $\# 3$ and smart net meter\#3. 


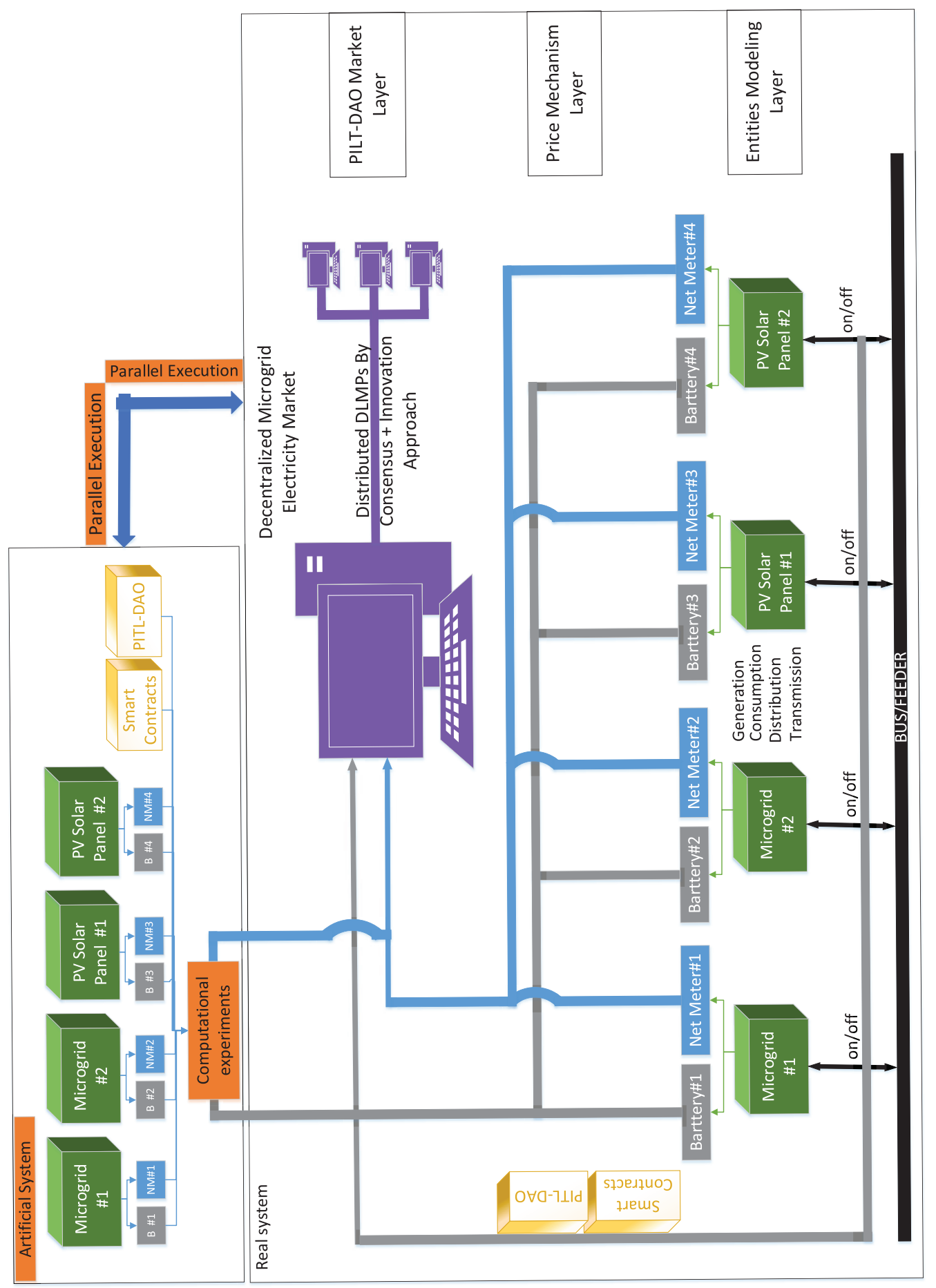

Figure 5.1: Whole PILT-DAO layers blueprint 
In the second layer, the price mechanism layer, the consensus + innovation approach is used to achieve DCOPF and distributed DLMPs calculation. And then, the most important is to program the smart contracts on the Ethereum constituting an ILT-DAO. The power generation and price values from this algorithm will be written in smart contracts and be sent to blockchain using Application Programming Interfaces (APIs) as well. This function will be shown in the following case study.

Finally, the ACP method is used to implement the PILT-DAO from ILT-DAO with the parallel system in the PILT-DAO market layer. The advantages to utilize the parallel system for ILT-DAO are: 1) Virtual programs can be fed-back for real; and 2) To protect and speed up real system data and calculation. The flowchart of detailed parallel execution process in PILT-DAO system is shown in Figure 5.2 [38].

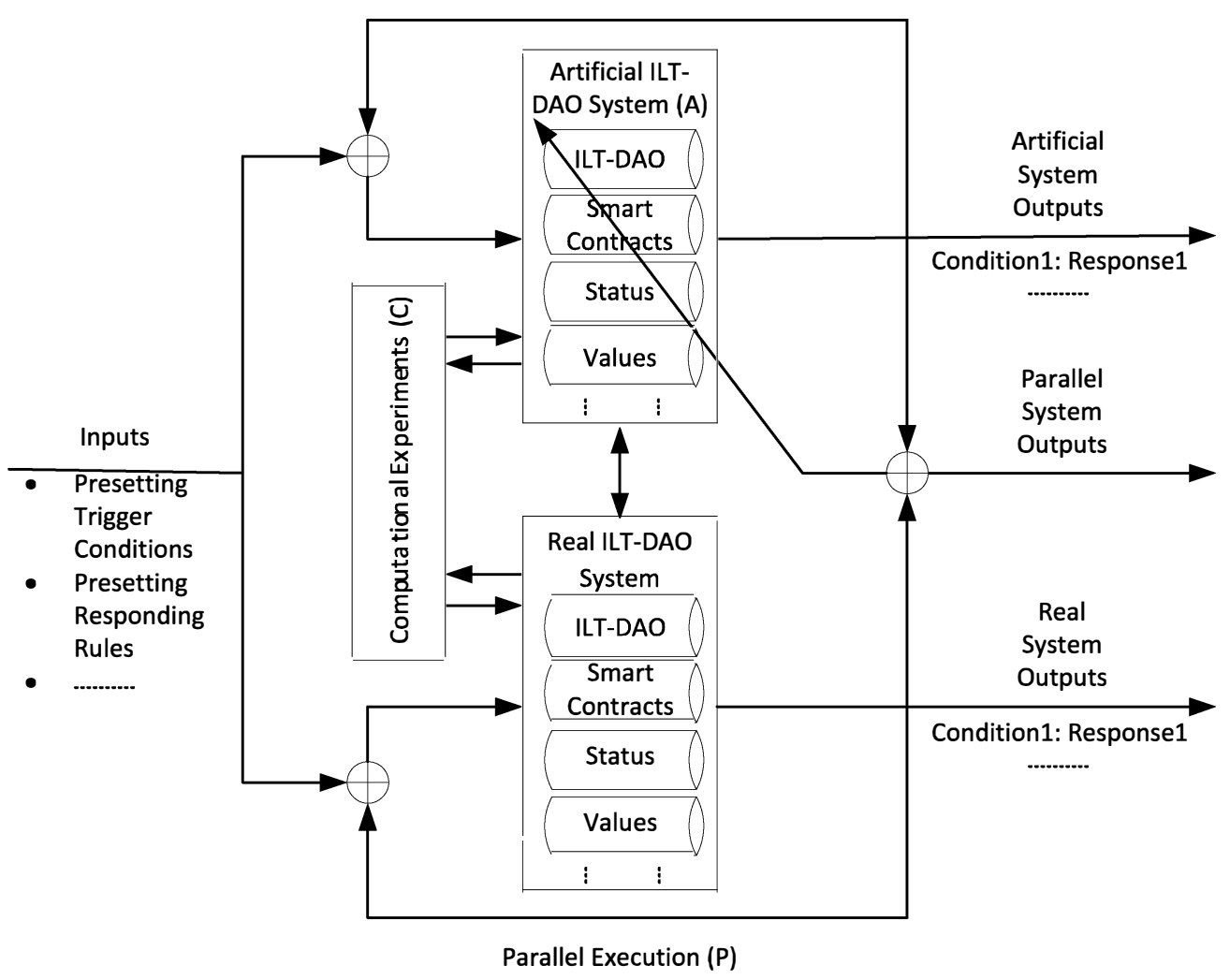

Figure 5.2: The flowchart of detailed parallel execution process in PILT-DAO 


\subsection{Case Study}

A nodejs case study named Distribution Power Trading (DPT) platform will be shown in this section. The distributed power trading system architecture is shown in Figure 5.3. Blockchain server is a serve to access Ethereum private chain and get the database of blocks, transactions and smart contracts, etc.. Distributed DLMPs server is to read MATLAB OPF results calculated by consensus + innovation algorithm. Contract server connects the database between blockchain server and distributed DLMPs server to finish transactions. The three servers are controlled by nodejs controller, and then, show them on web localhost:3000.

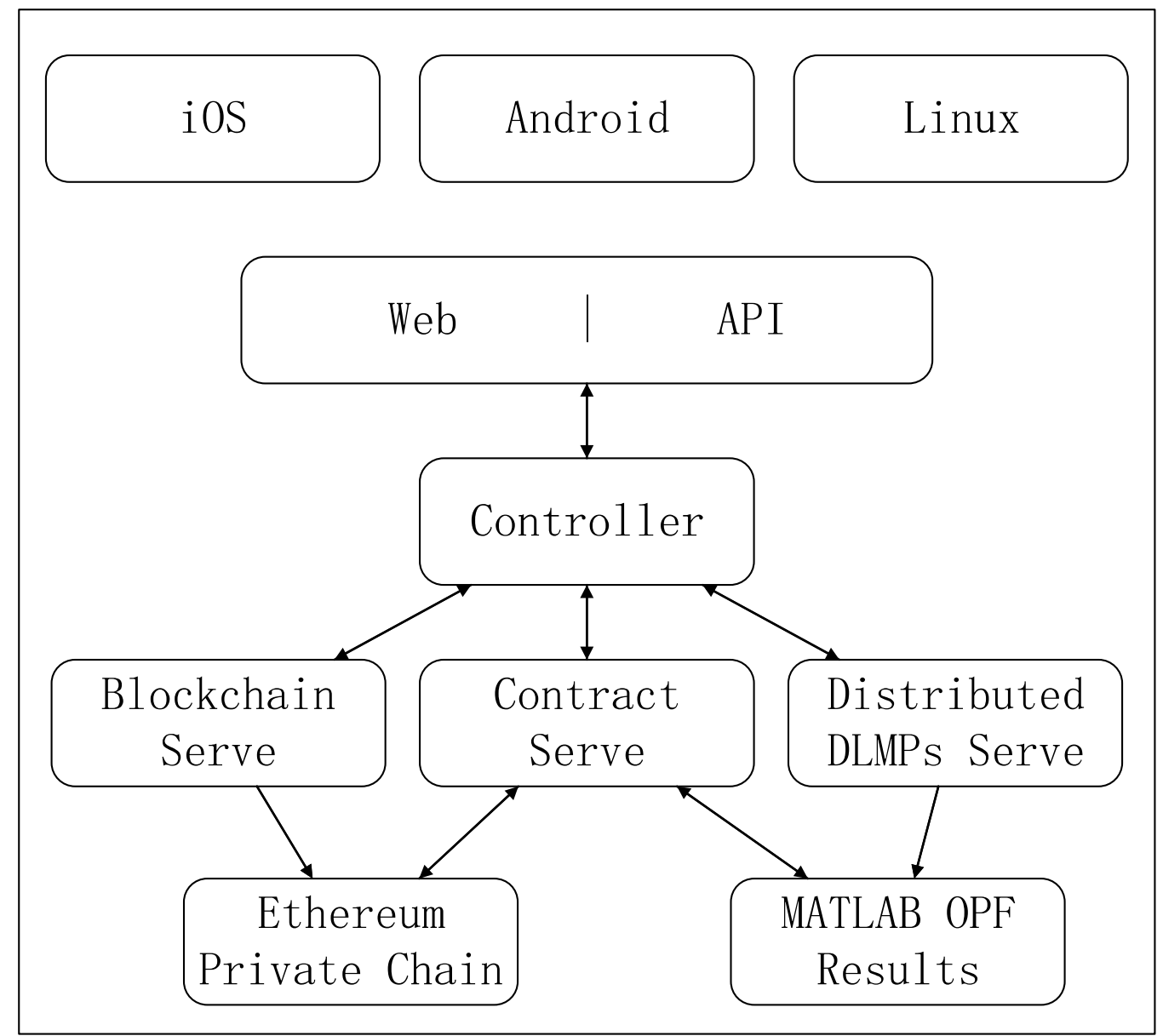

Figure 5.3: Distributed power trading system architecture 
The Ethereum private chain is created by GO language and Geth commands. The results of distributed DLMPs and units power generation from MATLAB algorithm are given in Figure 4.3 to 4.10 and sent to servers.

In servers, we use module-view-controller approach to create the user interface on the web localhost:3000. The home page of the user interface is shown in Figure 5.4 to show the account. At the beginning, the balance is 0 except Miner account. The Blocks and transactions information are shown in the block page Figure 5.5. Height means the number of current block. Hash is the current block transaction hash. When Tx is 0 , there is no transaction. For example, Tx is 2 at Height 23. That means there are 2 traded transactions. And, the two transactions details are shown in transaction page, Figure 5.6, including height, hash and time-stamp.Finally, the transfer page is a page to send a transaction from one account to another one, as in Figure 5.7. The price information is from MATLAB DLMPs such as Figure 4.5, $4.6,4.7$ and 4.9 .

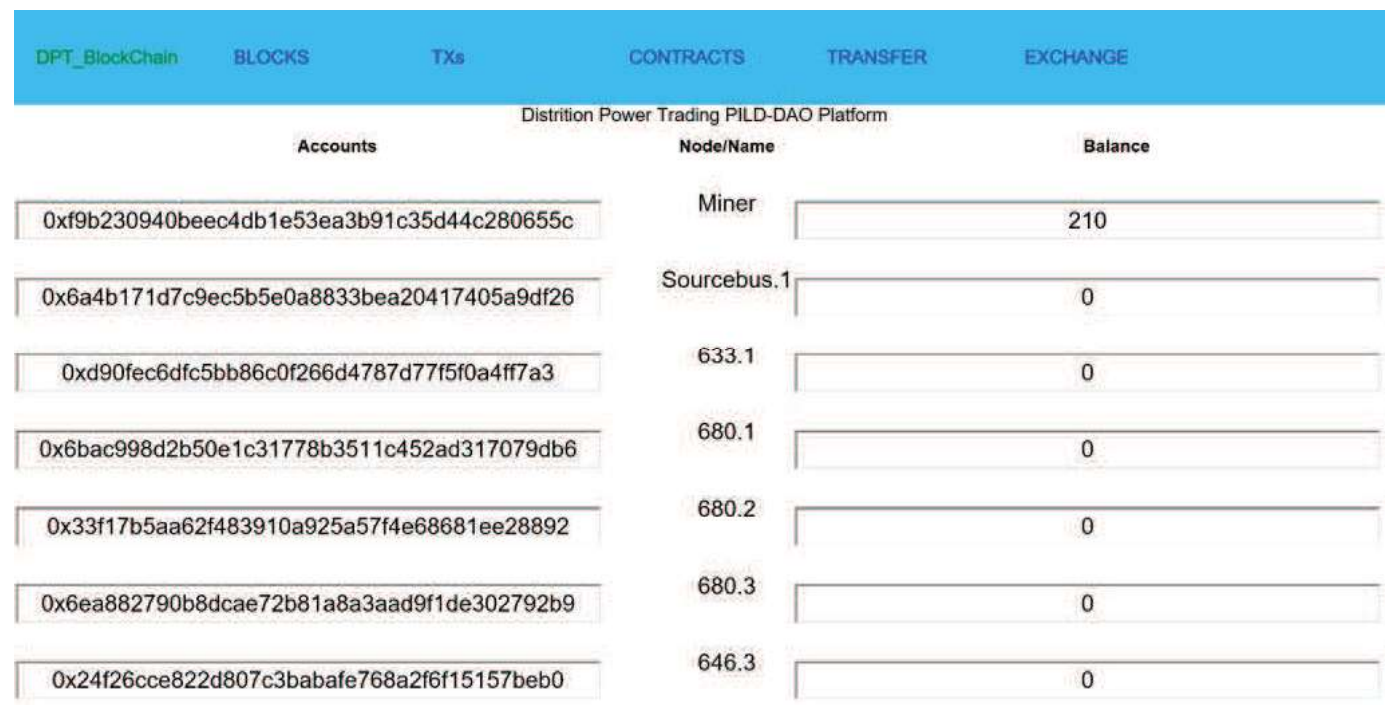

Figure 5.4: The home page of distribution power trading platform 


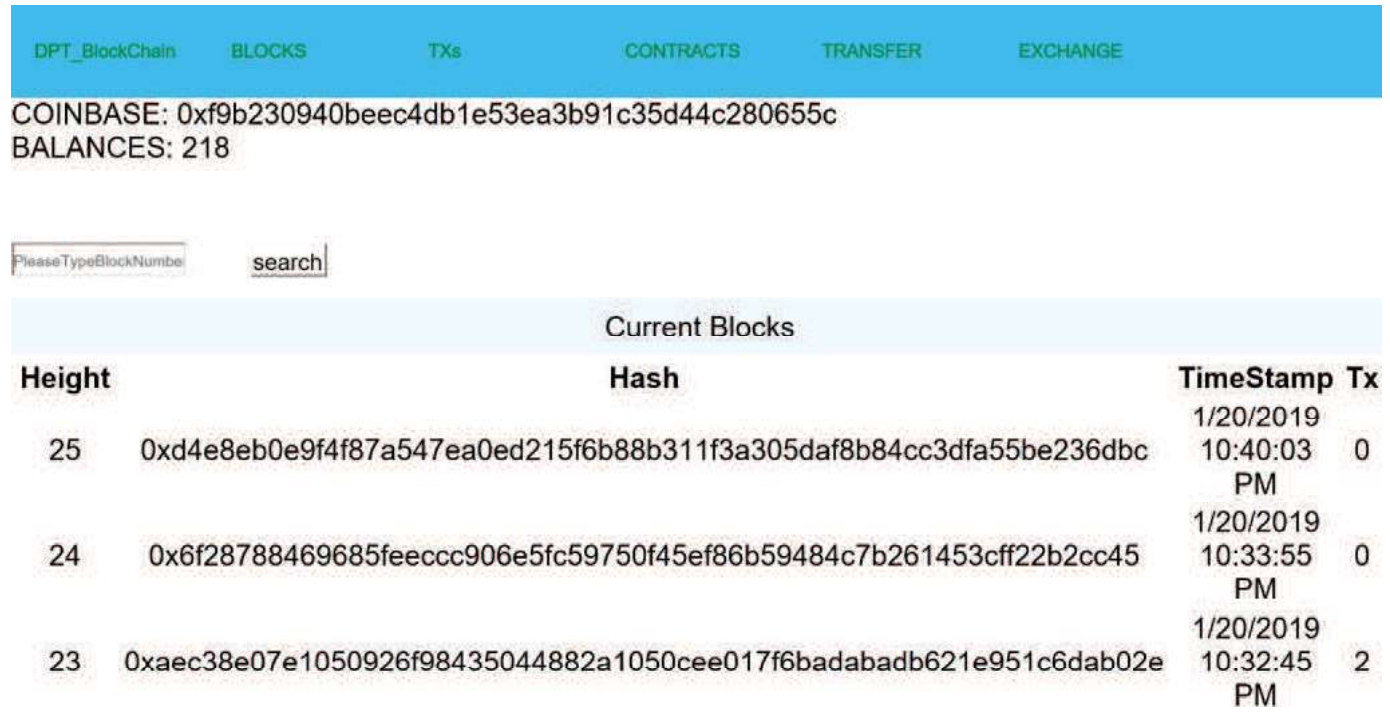

Figure 5.5: The block page of distribution power trading platform

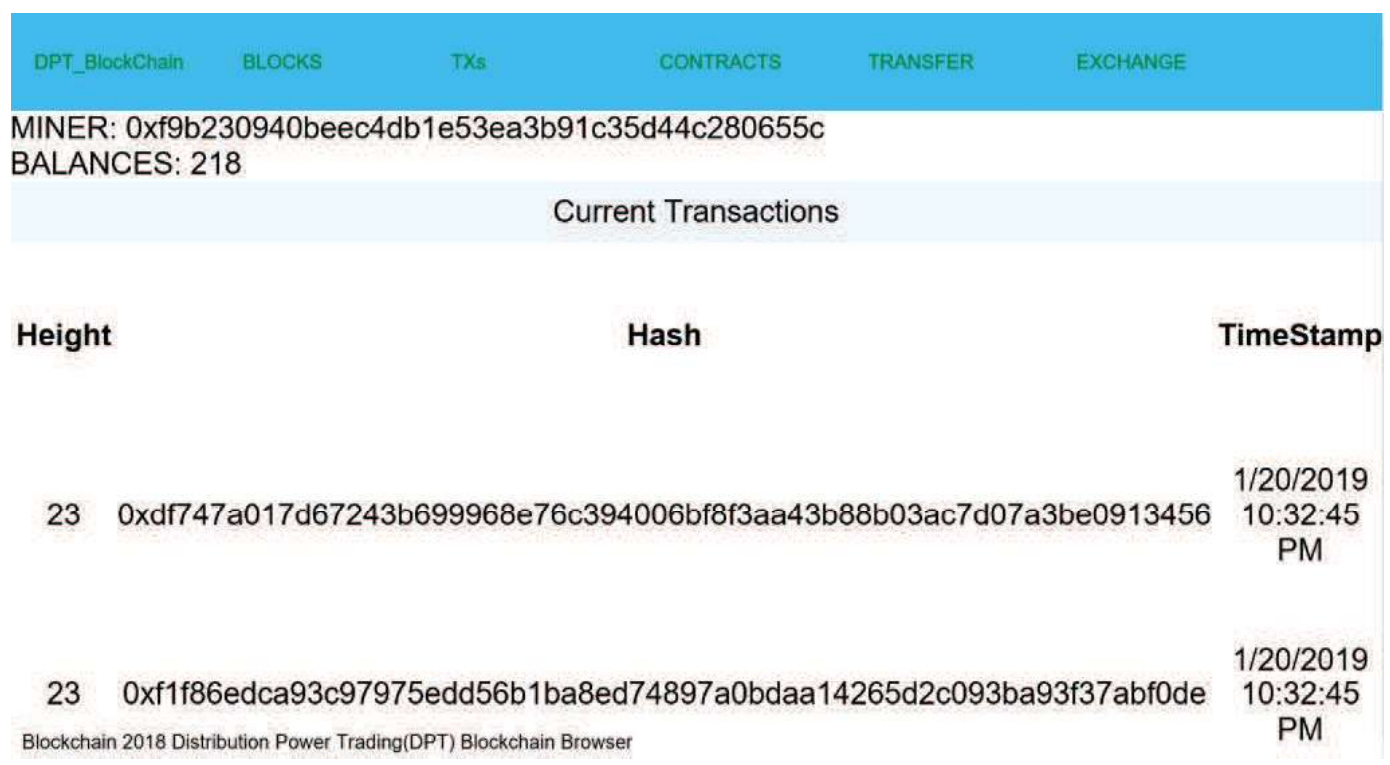

Figure 5.6: The transaction page of distribution power trading platform 


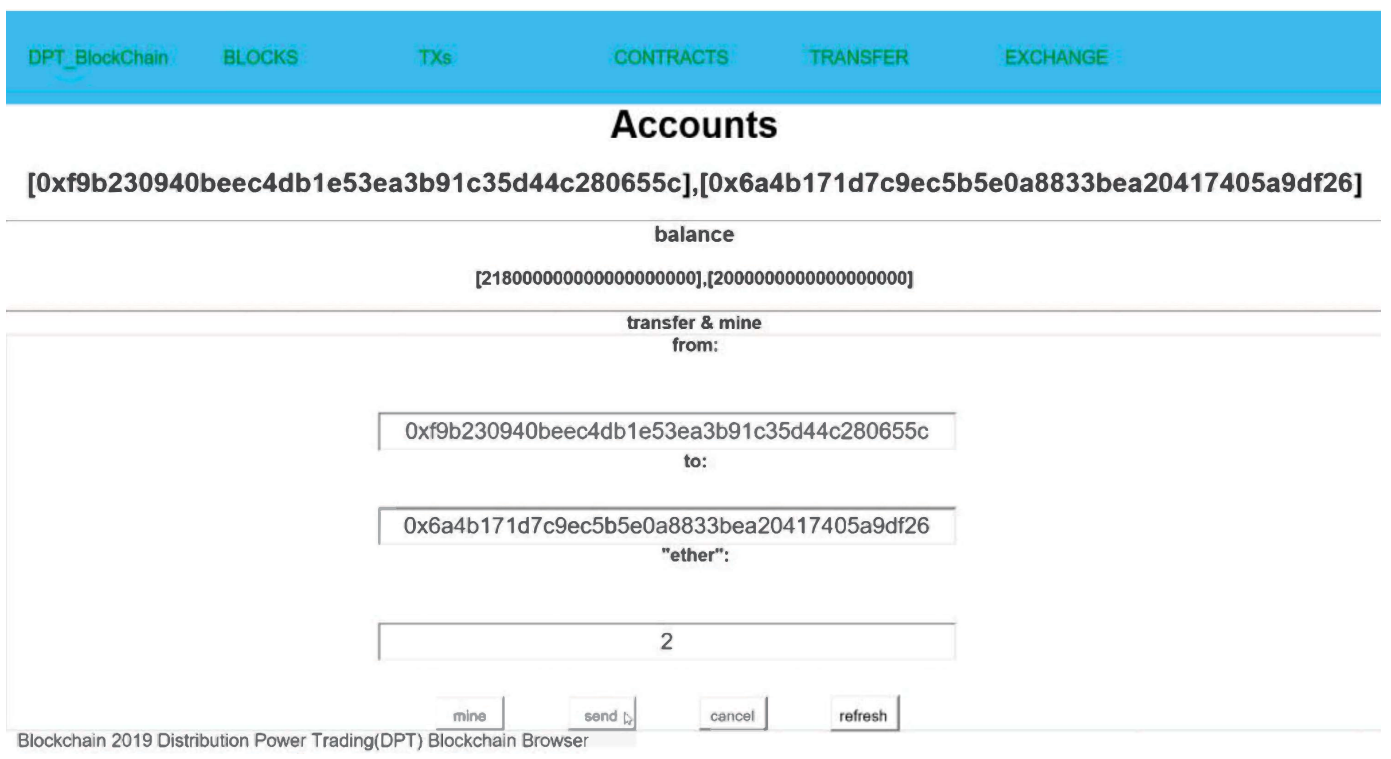

Figure 5.7: The transaction page of Ethereum Geth platform

After sending two transactions from miner to the accounts of Sourcebus. 1 and 680.1, the transactions information in Ethereum Geth platform is shown in Figure 5.8. We can see the blockHash number, block number, from account, gas price, to account, hash number and value (value unit is "Wei", 1 "eth" $=1 \times 10^{18}$ ) "Wei") etc.. And then, after miner mining the transaction, the balance of accounts changed also. The new balances of accounts is demonstrated in Figure 5.9. Node Sourcebus.1 ("0x6a4b171d7c9ec5b5e0a8833bea20417405a9df26") changed from 0 to 2. Node 684.1 ("0x59d20f8dd80268e3c4bdbdf87c947a4fc63bb43d") changed from 0 to 5 . 


\section{eth. getTransaction (' $0 x d f 747 a 017 d 67243 b 699968 \mathrm{e} 76 c 394006 b+8 f 3 a a 43 b 88 b 03 a c 7 d 07 a 3 b e 0913456$ )}

blockHash: "Gxaec38e07e1050926f98435044882a1050cee017f6badabadb621e951c6dabo2e",

blockNumber:

from: "Oxf9b230940beec4dbie53ea3b91c35d44c286655c",

gas:

gasPrice:

hash: "Oxdf747a017d67243b699968e76c394806bf843aa43b88b03ac7de7a3beg913456",

input: " $6 x "$,

nonce: 6 ,

r: "Ox738́e6fb23f4edb2e22ac28e $5 d 107 f f 499 b f c 150276 c 82 c e 38531449735209$ efd",

s: "6x3a1f8ef2dc952d4ae2e51 cecc12d7d2fca7be5746e344abf4b424126aabega4f",

to: "Ox6a4b171d7c9ec565e9a8833bea20417405a9df26",

transactionIndex:

v: "roxic",

value:

eth. getTransaction( ' $0 x f 1 f 86 e d c a 93$ c97975edd56b1ba8ed74897a0bdaa14265d2c093ba93f37abf0de ')

blockHash: "Oxaec 38e07e1050926f98435844882a1050cee617f6badabadb621e951c6dab82e",

blockNumber:

from: "exf9b2309.49beec4db1e53ea3b91c35d44c286655c",

gas:

gasPrice:

hash: "Oxf1f86edca93 c97975edd56b1babed74897abbdaa14265d2co93ba93f37abfode",

input: "6x",

nonce:

r: "Bxf310d6cb7561b6566e948951 c74d17fff7a6343551190565fdes811a89a1d9a7",

s: "0x437457d7c946adffgfef36781d89ef662 fda55ad73dab44e1.27318d5e4daf9e2",

to: "Bx59d20f8dd83268e3c4bdbdf87c947a4fc63bb43d",

transactionIndex:

v: $9 \times 1 b^{4}$,

value:

Figure 5.8: The transactions information in Ethereum Geth platform

\begin{tabular}{|c|c|c|c|}
\hline DPT_BlockChain: & CONTRACTS & TRANBFER. & EXCHANGE \\
\hline \multicolumn{4}{|c|}{ Distrition Power Trading PILD-DAO Platform. } \\
\hline Accounts & Node/Name & & Balance \\
\hline 0xf9b230940beec4db1e53ea3b91c35d44c280655c & Miner & & 218 \\
\hline 0x6a4b171d7c9ec5b5e0a8833bea20417405a9df26 & Sourcebus. 1 & & 2 \\
\hline 0xd90fec6dfc5bb86c0f266d4787d77ff5f0a4ff7a3 & 633.1 & & 0 \\
\hline 0x6bac998d2b50e1c31778b3511c452ad317079db6 & 680.1 & & 5 \\
\hline 0x33f17b5aa62f483910a925a57f4e68681ee28892 & 680.2 & & 0 \\
\hline 0x6ea882790b8dcae72b81a8a3aad9f1de302792b9 & 680.3 & & 0 \\
\hline $0 \times 24 f 26 c c e 822 d 807 c 3$ babafe $768 a 2 f 6 f 15157 b e b 0$ & 646.3 & & 0 \\
\hline
\end{tabular}

Figure 5.9: The home page of distribution power trading platform after transactions 


\section{Chapter 6}

\section{Conclusion and Future Works}

In conclusion, the three layers in the new architecture of small-scale microgrid energy market based on PILT-DAO are introduced in chapter 3, 4, and 5 respectively. Entities Modeling Layer adds 3 PVs and 2 MGs in the modified IEEE 13 node test feeder system topology. In the price mechanism layer, the consensus + innovations approach calculates DLMPs for one normal case and one contingency case. Blockchain technology using in Ethereum platform connects consensus + innovations algorithm in PILT-DAO market layer. In addition, a case study of small-scale microgrid energy market based on PILT-DAO is presented in chapter 5 in Figure 5.4 to 5.9. The research done in this thesis demonstrates that the new market structure can reduce the operation cost and increase the transaction security and transparency between DG suppliers with PILT-DAO blockchain technology.

However, several aspects of research are still needed in the future. They are:

1) Calculate the DLMPs under linearized ACOPF constraints using the distributed consensus + innovation algorithm;

2) Apply a new distributed algorithm to ED, DCOPF, and ACOPF;

3) Improve the user interface of distribution power trading based on PILT-DAO;

4) Study parallel modeling method. 


\section{Bibliography}

[1] E. lbanez A. Florita M. Heaney B.-M. Hodge M. Hummon and G. Stark D. Lew, G. Brinkman and G. Stark. The western wind and solar integration study. Technical Report NREL/TP-5500-55588, NREL, NREL, GOLDEN, CO, September 2013.

[2] David Palchak Joshua Novacheck Jack King Clayton Barrows Eduardo Ibanez Matthew OConnell Gary Jordan Billy Roberts Caroline Draxl Aaron Bloom, Aaron Townsend and Kenny Gruchalla. Eastern renewable generation integration study. Technical Report NREL/TP-6A20-64472, NREL, RePPAE LLC, GE, August 2016.

[3] Prepared by, Mathew J. Morey, and Laurence D. Kirsch. Retail choice in electricity: What have we learned in 20 years. 2266 .

[4] Esther Mengelkamp, Johannes Grttner, Kerstin Rock, Scott Kessler, Lawrence Orsini, and Christof Weinhardt. Designing microgrid energy markets. Applied Energy, 210:870-880, jan 2018.

[5] December. Distributed energy resources market design concept proposal a report by the new york independent system operator. 2017.

[6] Jarosław Stańczak, Weronika Radziszewska, and Zbigniew Nahorski. Dynamic pricing and balancing mechanism for a microgrid electricity market. In $A d$ vances in Intelligent Systems and Computing, pages 793-806. Springer International Publishing, 2015. 
[7] Antonio J. Conejo, Juan M. Morales, and Luis Baringo. Real-time demand response model. IEEE Transactions on Smart Grid, 1(3):236-242, dec 2010.

[8] Jian Wang, Qianggang Wang, Niancheng Zhou, and Yuan Chi. A novel electricity transaction mode of microgrids based on blockchain and continuous double auction. Energies, 10(12):1971, nov 2017.

[9] Kerstin Rock Scott Kessler Lawrence Orsini Christof Weinhardt Esther Mengelkamp, Johannes Garttner. Designing microgrid energy markets a case study: The brooklyn microgrid. 2017.

[10] Ahmad F. Taha Shen Wang and Jianhui Wang. Blockchain-assisted crowdsourced energy systems. In IEEE Power 6 Energy Society General Meeting 2018, Portland, Oregon, August 2018. IEEE.

[11] Qiuyu Peng and Steven H. Low. Optimal branch exchange for feeder reconfiguration in distribution networks. In 52nd IEEE Conference on Decision and Control, Florence, Italy, December 2013.

[12] Satoshi Nakamoto. Bitcoin: A peer-to-peer electronic cash system, 2009.

[13] A NEXT, GENERATION SMART, CONTRACT, DECENTRALIZED APPLICATION, PLATFORM, By Vitalik, and Buterin. Ethereum white paper. 2009.

[14] Vitalik Buterin. A next-generation smart contract and decentralized application platform. Technical report, Ethereum, 2017.

[15] Vitalik Buterin. Daos, dacs, das and more: An incomplete terminology guide. Technical report, Ethereum Blog, 2014. 
[16] F. Wang. Toward a paradigm shift in social computing: The acp approach. IEEE Intelligent Systems, 22(5):65-67, September 2007.

[17] Fei-Yue Wang. Cpscom 2010 keynote speech: Feiyue wang. In 2010 IEEE/ACM Int'l Conference on Green Computing and Communications Int'l Conference on Cyber, Physical and Social Computing, pages lvi-lvi, December 2010.

[18] Nick Szabo. Smart contracts. 1994.

[19] by Ralph C. Merkle and merkle@merkle.com. Daos, democracy and governance. 2016.

[20] Huaiguang Jiang, Yingchen Zhang, Eduard Muljadi, Jun Zhang, and Wenzhong Gao. A short-term and high-resolution distribution system load forecasting approach using support vector regression with hybrid parameters optimization. IEEE Transactions on Smart Grid, 2016.

[21] Huaiguang Jiang, Xiaoxiao Dai, David Wenzhong Gao, Jun Jason Zhang, Yingchen Zhang, and Eduard Muljadi. Spatial-temporal synchrophasor data characterization and analytics in smart grid fault detection, identification, and impact causal analysis. IEEE Transactions on Smart Grid, 7(5):2525-2536, 2016 .

[22] Huaiguang Jiang, Yingchen Zhang, Jun Jason Zhang, David Wenzhong Gao, and Eduard Muljadi. Synchrophasor-based auxiliary controller to enhance the voltage stability of a distribution system with high renewable energy penetration. IEEE Transactions on Smart Grid, 6(4):2107-2115, 2015.

[23] Yi Gu, Huaiguang Jiang, Yingchen Zhang, Jun Jason Zhang, Tianlu Gao, and Eduard Muljadi. Knowledge discovery for smart grid operation, control, and situation awarenessa big data visualization platform. In 2016 North American Power Symposium (NAPS), pages 1-6. IEEE, 2016. 
[24] J. J. Zhang, F. Wang, Q. Wang, D. Hao, X. Yang, D. W. Gao, X. Zhao, and Y. Zhang. Parallel dispatch: a new paradigm of electrical power system dispatch. IEEE/CAA Journal of Automatica Sinica, 5(1):311-319, January 2018.

[25] Jun Jason Zhang, David Wenzhong Gao, Yingchen Zhang, Xiao Wang, Xiangyang Zhao, Dongliang Duan, Xiaoxiao Dai, Jun Hao, and Fei-Yue Wang. Social energy: mining energy from the society. IEEE/CAA Journal of Automatica Sinica, 4(3):466-482, 2017.

[26] J. Hao, X. Dai, Y. Zhang, J. Zhang, and W. Gao. Distribution locational realtime pricing based smart building control and management. In 2016 North American Power Symposium (NAPS), pages 1-6, September 2016.

[27] Jian Qiu and Yun-Bo Yi. Random walk simulation model of diffusion in circular and elliptical particulate composites. International Journal for Multiscale Computational Engineering, 16(2), 2018.

[28] J Qiu, YB Yi, and X Guo. Computational prediction of electrical and thermal conductivities of disklike particulate composites. International Journal of Computational Materials Science and Engineering, 4(03):1550013, 2015.

[29] Jian Qiu. Computational Modeling of Percolation Conduction and Diffusion of Heterogeneous Materials. PhD thesis, University of Denver, 2017.

[30] Yingchen Zhang Rui Yang. Three-phase ac optimal power flow based distribution locational marginal price. In 2017 IEEE Power \& Energy Society Innovative Smart Grid Technologies Conference (ISGT), number 17315158, Washington, DC, USA, April 2017. IEEE. 
[31] Yingchen Zhang Rui Yang. Three-phase ac optimal power flow based distribution locational marginal price: Preprint. Technical Report NREL/CP-5D0068112, NREL, NREL, GOLDEN, CO, May 2017.

[32] A. Khodaei. Resiliency-oriented microgrid optimal scheduling. IEEE Transactions on Smart Grid, 5(4):1584-1591, July 2014.

[33] K. P. Schneider, B. A. Mather, B. C. Pal, C. . Ten, G. J. Shirek, H. Zhu, J. C. Fuller, J. L. R. Pereira, L. F. Ochoa, L. R. de Araujo, R. C. Dugan, S. Matthias, S. Paudyal, T. E. McDermott, and W. Kersting. Analytic considerations and design basis for the ieee distribution test feeders. IEEE Transactions on Power Systems, 33(3):3181-3188, May 2018.

[34] G. Hug, S. Kar, and C. Wu. Consensus + innovations approach for distributed multiagent coordination in a microgrid. IEEE Transactions on Smart Grid, 6(4):1893-1903, July 2015.

[35] J. Mohammadi, G. Hug, and S. Kar. Asynchronous distributed approach for dc optimal power flow. 2015 IEEE Eindhoven PowerTech, pages 1-6, June 2015.

[36] J. Mohammadi, G. Hug, and S. Kar. Fully distributed dc-opf approach for power flow control. In 2015 IEEE Power Energy Society General Meeting, pages $1-5,2015$.

[37] Gabriela Hug Javad Mohammadi, Soummya Kar. Distributed approach for dc optimal power flow calculations. arXiv: Cornell University, pages 1-11, October 2014. Optimization and Control.

[38] Zhi-Wei Gao and Fei-Yue Wang. New results on doubly coprime fractional representations of generalized dynamical systems. IEEE Transactions on Automatic Control, 48(2):299-303, February 2003. 


\section{Appendix A}

\section{ACRONYMS}

ACP

API

CPS

CPSS

CRS

DAO

DER

DG

DLMP

DPT

DS

DSO

ED

EMS
Artificial systems, Computational experiments, Parallel execution

Application Programming Interface

Cyber-Physical System

Cyber-Physical-Social System

Congressional Research Service

Decentralized Autonomous Organization

Distributed Energy Resource

Distributed Generation

Distribution Locational Marginal Price

Distribution Power Trading

Distributed Storages

Distributed System Operator

Economic Dispatch

Energy Management System 


\begin{tabular}{|c|c|}
\hline EVM & Ethereum Virtual Machine \\
\hline FERC & Federal Energy Regulatory Commission \\
\hline ILT & Integrity, Longevity, and Transparency \\
\hline ISO & Independent System Operator \\
\hline LMP & Locational Marginal Price \\
\hline MCP & Market clearing Price \\
\hline MG & Microgrid \\
\hline MW & Megawatt \\
\hline OPF & Optimal Power Flow \\
\hline $\mathbf{P F}$ & Power Flow \\
\hline P2P & Peer-to-Peer \\
\hline PILT & Parallel, Integrity, Longevity, and Transparency \\
\hline PJM & Pennsylvania, New Jersey, Maryland \\
\hline P.U. & Per Unit \\
\hline PoW & Proof-of-Work \\
\hline RTO & Regional Transmission Organization \\
\hline
\end{tabular}




\section{Appendix B}

\section{Nomenclature}

Functions:

$\mathscr{F}$

Sets:

$\Omega_{G}$

$\Omega_{N}$

$\Omega_{k}$

$\Omega_{L}$

Indices:

$n$

$t$

$i$

$k$

j
Operation Cost Function

Set of Generators

Set of Nodes

Set of Buses

Set of Line Segments

Index of Generators

Index of time

Index of iteration

Index of buses $\mathrm{k}$

Index of buses $\mathrm{j}$ 
Binary and Integer Variables:

$\begin{array}{ll}X_{i j} & \text { Line Reactance } \\ \bar{P}_{i j} & \text { Maxmium Power Flow } \\ -\underline{P}_{i j} & \text { Minmium Power Flow }\end{array}$

